\title{
Distortion of a poly-crystalline Al bar in a vice fixture: Molecular dynamics analysis of grain movement and rotation
}

Vardan Hoviki Vardanyan

University Kaiserslautern

Barbara S Linke

University of California Davis

Herbert M Urbassek ( $\square$ urbassek@rhrk.uni-kl.de )

University Kaiserslautern https://orcid.org/0000-0002-7739-4453

\section{Research Article}

Keywords: nanofabrication, distortion, plasticity, grain boundary, grain rotation, molecular dynamics

Posted Date: May 3rd, 2021

DOl: https://doi.org/10.21203/rs.3.rs-331395/v1

License: (c) (1) This work is licensed under a Creative Commons Attribution 4.0 International License.

Read Full License

Version of Record: A version of this preprint was published at The International Journal of Advanced Manufacturing Technology on July 27th, 2021. See the published version at https://doi.org/10.1007/s00170-021-07641-y. 


\title{
Distortion of a poly-crystalline Al bar in a vice fixture: Molecular dynamics analysis of grain movement and rotation
}

\author{
Vardan Hoviki Vardanyan, ${ }^{1}$ Barbara S. Linke, ${ }^{2}$ and Herbert M. Urbassek ${ }^{1, *}$ \\ ${ }^{1}$ Physics Department and Research Center OPTIMAS, University Kaiserslautern, \\ Erwin-Schrödinger-Straße, D-67663 Kaiserslautern, Germany \\ ${ }^{2}$ University of California Davis, Mechanical and Aerospace Engineering Department, 1 Shields Ave, Davis, CA 95616, USA
}

(Dated: March 16, 2021)

\begin{abstract}
The deformation of a nano-sized polycrystalline Al bar under the action of vice plates is studied using molecular dynamics simulation. Two grain sizes are considered; deformation in the fine-grained sample is mainly caused by grain-boundary processes (sliding and rotation), while dislocation plasticity dominates in the coarse-grained sample. We show that the sample distortion is reflected by the center-of-mass motion of the grains. Grain rotation is responsible for surface roughening after the loading process. While the plastic deformation is caused by the loading process, grain rearrangements under load release also contribute considerably to the final sample distortion.
\end{abstract}

Keywords: nanofabrication, distortion, plasticity, grain boundary, grain rotation, molecular dynamics

\section{INTRODUCTION}

The plastic deformation of polycrystalline metal samples is a topic of long-standing interest in materials science [1]. It is of immediate importance in the field of mechanical engineering, where plastic deformation processes accompany more or less every treatment of a workpiece, leading both to residual stresses and to workpiece distortions [2]. The origin of such distortions is the topic of actual scientific research [3-7].

While machining processes on the microscale are commonly used in ultra-precision engineering [8], nanometersized mechanisms are out of reach for most applications. However, in the fields of photolithography, chemical etching, laser machining, atomic force lithography, or focused ion beam lithography, nanofabrication techniques are already being used [9]. It is therefore of interest to widen our understanding of plastic processes by including processes occurring in nanofabrication. Here, atomistic simulation methods based on molecular dynamics simulation, have proven useful $[10,11]$. The behavior of samples under homogeneous strain has been investigated best [12-19]. Also several machining processes have been studied, including nanoindentation [20,21], scratching [22] and nanocutting [23] of surfaces. Several of the results obtained also allowed extension to larger spatial scales, i.e., to $\mu \mathrm{m}$-sized samples, for instance the generation of dislocations [21] or the effect of the tip geometry on the plastic processes [22].

On the nanoscale, distortion and the generation of residual stresses are based on the plastic processes occurring in the material under mechanical loading. In many metals, plasticity is dominated by dislocations which are activated during loading [1, 24]. In nano-crystalline material, in addition grainboundary-based processes - in particular grain-boundary sliding and rotation - contribute to the plastic deformation [25-

\footnotetext{
* Corresponding author: Email: urbassek@rhrk.uni-kl.de; http://www.physik.uni-kl.de/urbassek/
}

28]. The balance of these processes for describing distortion has to our knowledge not been investigated up to now.

In the present paper, we will study the mechanical deformation of an $\mathrm{Al}$ bar which is compressed in an inhomogeneous way by vice plates [29], see Fig. 1a. Besides the plastic deformation processes, we investigate in particular the processes occurring on the individual grains, i.e., grain sliding and rotation, and the eventual distortion of the bar, which is the externally measurable result of the plastic processes occurring inside the bar.

\section{METHOD}

We study an Al block, see Fig. 1a, with sizes of $80 \mathrm{~nm}$ $\times 40 \mathrm{~nm} \times 60 \mathrm{~nm}$ in $x, y$ and $z$ directions, consisting of approximately $12 \times 10^{6}$ atoms. The block is subject to periodic boundary conditions in $y$ direction; the other surfaces are free. The bottom half of the $x-z$ surfaces are held by rigid vice plates. In the course of the simulation, these plates move inward along the $x$ direction with a velocity of $10 \mathrm{~m} / \mathrm{s}$ for 200 ps, compressing the $\mathrm{Al}$ bar from 80 to $78 \mathrm{~nm}$; then the fixtures are held for 200 ps to allow the sample to relax; and finally the vices are released by moving with $10 \mathrm{~m} / \mathrm{s}$ to their initial positions.

In a previous publication [29], this procedure was applied to a single-crystalline Al block where all surfaces were oriented in $\{100\}$ directions. In the present work, we use polycrystalline $\mathrm{Al}$ blocks. In order to understand the effect of grain size, we use two samples: a fine-grained sample consisting of 180 grains with an average size of $10 \mathrm{~nm}$, and a coarsegrained sample with 18 grains of $22 \mathrm{~nm}$ size; these samples are depicted in Fig. 1b. They are generated using Voronoi tesselation [30-32]. For the preparation of relaxed samples, we proceed as follows. First, we heat the samples to $500 \mathrm{~K}$ during $50 \mathrm{ps}$ in order to relax the grain boundaries in the samples; then we keep the temperature constant for another $50 \mathrm{ps}$, and finally decrease the temperature to $300 \mathrm{~K}$ during $50 \mathrm{ps}$. In a final step, the samples are relaxed for $250 \mathrm{ps}$ at $300 \mathrm{~K}$ in an 
isothermal-isobaric NPT ensemble such that all components of the stress tensor decrease to zero. During the compression and release simulations, the atoms in a stripe of width 1 $\mathrm{nm}$ in the middle between the vice plates are subjected to a Nose-Hoover thermostat to keep the temperature at $300 \mathrm{~K}$. In addition, the lower part of this stripe with a height of $1 \mathrm{~nm}$ has been fixed in order to prevent rigid-body translation and rotation of the entire sample during the release phase.

The vice plates have a thickness of $2 \mathrm{~nm}$ and a height of $34 \mathrm{~nm}$. They consist of around 500,000 carbon atoms each, arranged in a rigid diamond-lattice structure.

The $\mathrm{Al}$ atoms interact via the Mendelev et al. potential [33] with each other. The Al-carbon interaction is modeled by a purely repulsive potential obtained from a Lennard-Jones potential cut off at its minimum [29].

The simulations are performed with the software LAMMPS [34] and the results are analyzed and visualized using OVITO [35].

\section{RESULTS}

\section{A. Forces}

The force on the vices during the pressing, holding and release phases of the simulation are shown in Fig. 2. We show the forces acting on the vice plates on the left- and right-handside, in order to be able to discuss the asymmetry caused by grain sliding and rotation, see Sects. III D and III E below.

During the pressing phase, initially the force increases in proportion to the distance moved by the plates; however, in the second half of the pressing phase, the forces saturate and even start decreasing. This happens since the yield stress of the material has been reached. The average pressure reached can be estimated as $p=F / A$; with the force maximum $F=3$ $\mu \mathrm{N}$ and the area of the vice plates of $A=1200 \mathrm{~nm}^{2}$, we have $p=2.5 \mathrm{GPa}$. This value may be compared to the hardness of single-crystalline $\mathrm{Al}$ in this potential of 6 GPA [36]. The yield stress in nanocrystalline samples has been found to be smaller and is in the range of 1.6 to $3.5 \mathrm{GPa}$, varying with grain size $[14,37-41]$. We note that these values are considerably above the yield stress of macroscopic polycrystalline $\mathrm{Al}$ due to the Hall-Petch effect.

The yield mechanisms in the coarse- and fine-grained samples are different $[39,42]$, see discussion in Sect. III B below, and hence the two samples have two different force maxima.

During the holding phase, the force decreases as the material relaxes internally by dislocation- and grain-boundarybased mechanisms, see Sect. III B below. Stress release is particularly pronounced for the coarse-grained sample, as here dislocations are more active; the dislocation activity is visible in the force evolution by the considerable fluctuations seen that originate from individual dislocation processes occurring in the sample. After around $100-150$ ps of holding time, the forces have reached their equilibrium values. The final pressure reached in the coarse-grained sample is only half of that in the pressing-phase and amounts to only $1.25 \mathrm{GPa}$.
During release of the vices, the force continuously decreases towards zero. The vices lose contact with the sam-

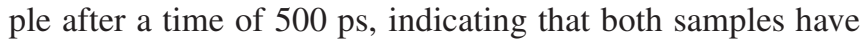
shrunk in $x$ direction by around $1 \mathrm{~nm}$ due to the plastic deformation in the entire compression and release operation.

We note that the strain rate used in our simulations amounts to $2.5 \times 10^{8} \mathrm{~s}^{-1}$. It has been shown $[43,44]$ that below strain rates of around $1 \times 10^{9} \mathrm{~s}^{-1}$, elastic and plastic properties - in particular the yield stress - depend only negligibly on strain rate. Above that critical strain rate, dislocation nucleation is prohibited.

\section{B. Deformation mechanism}

The deformation mechanisms of nanocrystalline materials are known to depend on grain size. In particular, it has been found [44] for the case of fcc $\mathrm{Cu}$ that above a grain size of around $15 \mathrm{~nm}$, deformation is governed by dislocation plasticity, which occurs inside grains, and below that grain size by grain-boundary processes, such as grain-boundary sliding and rotation. Dislocations nucleate in grains as a result of stress concentration in grain boundaries and relief of this stress in the grain interior for sufficiently large grains. While the quoted study [44] was on $\mathrm{Cu}$, the grain sizes chosen by us - 10 and $22 \mathrm{~nm}$ - lie clearly below and above the critical grain size, such that we expect to find these distinct behaviors also in our study on $\mathrm{Al}$.

In order to quantify the deformation processes both inside the grains and in the grain boundaries, we use the von-Mises shear strain (VMSS) that is readily evaluated for each atom in the sample [45]. Shear strain is the prime agent to nucleate and move dislocations, as well as to shape grain boundaries.

Focussing on the grain-boundary activity first, Fig. 3 shows the fraction of grain-boundary atoms with VMSS $>0.1$. The strain at the end of each phase (pressing, holding, and release) is calculated with respect to the configuration at the beginning of the phase. We see that in the fine-grained sample, in each phase more than half of the grain-boundary atoms have been considerably sheared, evidencing a high occurrence of grain-boundary mediated plastic deformation. The fraction of grain-boundary atoms that have been similarly sheared is considerably smaller for the coarse-grained sample. These numbers show that the above distinction made for nanocrystalline $\mathrm{Cu}$ also holds in our case of machined Al. Interestingly, the fraction of sheared grain-boundary atoms is largest under the release step. i.e., when the sample is allowed to return to a pressure-free state.

Apart from these fractions, the snapshots shown in Fig. 4 demonstrate the occurrence of dislocation-based and grainboundary-mediated processes in greater detail. In this view on the surface of the sample, dislocations inside the grains are marked and grain boundaries are colored according to whether their shear exceeded VMSS $=0.1$. First, it is clear that the number and length of dislocations is considerably higher in the coarse-grained sample. Second, the region where dislocations are nucleated extends towards the upper end of the vice plates and even above in the coarse-grained sample, while 
they are concentrated towards the lower surface in the finegrained sample. Not surprisingly, the largest amount of dislocation nucleation is found in the pressing phase, where a previously well-relaxed sample is quickly strained out of equilibrium. However, even the holding and release phases allow to produce novel dislocations or let already existing dislocations migrate. These findings corroborate our conclusion that dislocation plasticity is more readily found in the coarse-grained sample.

The role of grain-boundary activity can also be analyzed in Fig. 4. After pressing the samples, almost all grain boundaries in the bottom half of the sample are found to be strongly sheared, VMSS $>0.1$. Interestingly, the grains close to the vice plates are not as much sheared as those in the middle of the samples; this is due to the fact that close to the plates, the stress field is mostly uniaxial, and more shear is expected at the upper ends of the plates and in the middle of the sample. Again grain-boundary activities continue during the holding and the release phases, but are more prominent in the finegrained sample.

Besides shear strain, also hydrostatic pressure is an important indicator of the stress fields generated in the sample. It is shown in Fig. 5 in atomic resolution; pressure data are averaged over 0.5 ps to get rid of atomic vibrations. The plots give the same view as Fig. 4. As was noted above, the averaged (uniaxial) stress in $x$ direction amounts to $2.5 \mathrm{GPa}$ in the bottom half of the sample. The figure shows that even in the bottom half, the hydrostatic pressure is far from a homogeneous distribution; this is caused by the differing orientations of the grains. While at the end of the pressing phase, the grain interiors are all compressively strained, some regions - in particular where a high dislocation density has been produced are under tensile strain. Grain boundaries act as sources of high stress; some low-angle grain boundaries can be resolved in this figure as a chain of dislocations with their alternating compressive and tensile stress fields. Other grain boundaries are under primarily compressive stress.

During the holding phase, pressures equilibrate somewhat in the grains, as can be seen by a stronger mix of compressive and tensile stressed regions; this goes in line with the reduction of the forces needed to keep the vice plates in position, Fig. 2. After release, this equilibration process has further proceeded, since now the global pressure vanishes. Interestingly, stress release at grain boundaries is faster in the case of the fine-grained system.

The final snapshots in Fig. 5 show the stresses remaining in the sample after the entire process; they constitute the residual stresses. A comparison with Fig. 4 demonstrates that some of the stresses inside the grains may be attributed to the dislocations that have been produced there; other sources of high pressure are in the vicinity of triple junctions of grain boundaries. However, the inhomogeneity of the pressure distribution - which is under vanishing global pressure - is astonishing, since pressures vary between -1 and $+1 \mathrm{GPa}$.

\section{Surface roughness}

The snapshots, Fig. 4 and Fig. 5, show already strong deformations of the sample surface. During pressing and holding, the upper surface becomes curved in a convex way; this curvature disappears after release. The lower surface becomes rough, and this roughness does not disappear. This is due to the different grain orientations and the various grain rotations (and sliding processes). Another interesting feature is the leftright asymmetry, which develops for the coarse-grained sample in the pressing phase and remains through the holding and release phases and will be discussed in Sect. IIID and IIIE below.

We analyze the roughness developing in the bottom surface in greater detail in Fig. 6 which shows the height distribution of the bottom surface profile for the coarse-grained and finegrained samples. The average and the standard deviation of the distributions are assembled in Table 1 . We use the standard deviation of the height profile as a measure of surface roughness.

During pressing, the standard deviation increases by a factor of 4.5 (7) with respect to the original value for the coarsegrained (fine-grained) sample. During holding, the roughness increases even further, but reduces somewhat in the release phase. The reason for this surface roughness is grain sliding and rotation as will be discussed in the next two sections; these processes cannot be made undone during the release phase. The height profile of the coarse-grained sample, Fig. 6a, is far from a Gaussian profile as it exhibits two peaks in the height spectrum, which are caused by individual grains popping out of the original surface; this feature is even enhanced during the holding phase. There are 42 grains in the fine-grained sample at the bottom surface, but only 13 grains in the coarse-grained sample. Therefore, the multitude of grains contributing to the surface in the fine-grained sample randomize the height profile and lead to a distribution that is closer to a Gaussian.

\section{Center-of-mass grain translation}

In order to describe the translational motion (known as sliding) of grains, we study their center-of-mass displacements, $\vec{d}$, [25]. Since there is no standard analysis available for this purpose, we proceed as follows. We cut 7 slabs extending in the $x-z$ plane with a thickness of $2 \mathrm{~nm}$ in the $y$ direction, which are evenly distributed on the $y$ axis. In these slabs it is possible to analyze the center-of-mass translation of the grains in the $x-z$ plane, as we can neglect any motion in $y$ direction. We present the resulting translational motions as histograms in Fig. 7. This figure shows the displacements during the pressing, holding and release phases. In each phase, the displacement between the beginning and the end of the phase are shown. The total displacement, $d_{\mathrm{abs}}=|\vec{d}|$, and the components $d_{x}$ and $d_{z}$ along the $x$ and $z$ axes are shown.

Grain translation during the pressing phase, Fig. 7a, is best described by the component parallel to the force axis, $d_{x}$. The distributions shows two peaks at $-20 \AA$ and $+20 \AA$, corresponding to grains located close to the vice plates, which are 
forced to move the entire distance covered by the nearby vice. The majority of the grains, however, suffer only little motion, since they are located in the middle of the sample, between the two vices. Apart from this horizontal motion, a component $d_{z}$ in vertical direction is seen. The majority of the grains are moved upwards and thus contribute to the curvature of the upper surface seen in Fig. 6. In the pressing phase, no large difference between the coarse-grained and the fine-grained samples shows up.

Note that in a purely elastic compression, grain centers initially positioned at $x$ would move by a distance $(1-\varepsilon) x$, where $\varepsilon=0.05$ is the applied uniaxial strain. The $d_{x}$ data in Fig. 7a would thus follow a rectangular distribution of constant height. Our simulation results thus demonstrate the large deviation of plastic grain flow from this elastic reference case.

Considerable differences are observed during the holding phase, Fig. 7b. Here, grains in the coarse-grained sample are shifted to the right hand side, $d_{x}>0$, with an amplitude of 5 $\AA$. This shift corresponds to the left-right asymmetry shown in Fig. 4 and Fig. 5. The fine-grained sample also drifts further in horizontal direction in order to release the compressive strain present in the lower part of the sample; grain movements in the coarse-grained sample are larger in size, but show as much upwards as downwards motion.

The left-right asymmetry showing up here is caused by the individual grain movements. In the coarse-grained sample, an analysis of grain motion shows a relative movement of the upper part of the sample towards the right, accompanied by a downward motion of the grains in the vicinity of the righthand vice plate. The fine-grained sample does not show a corresponding asymmetry, since here, grain motion is more random.

Also during the release phase, considerable grain movements are observed, Fig. 7c. The curvature of the upper part of the sample is reduced, leading to a downward grain motion, $d_{z}<0$. While the grain motion in $x$ direction is symmetrical for the fine-grained sample, the coarse-grained sample tends to reduce the asymmetric motion that occurred during the holding phase, featuring an average $d_{x}<0$.

The asymmetry observed in the grain motion for the coarsegrained sample also shows up when considering the forces acting on the vices, Fig. 2. As the vices are compressed with constant velocity, individual grain movements oppose the vice motion with different forces. Interestingly, this asymmetry is particularly pronounced in the holding stage, but is also visible in the pressing and release stages.

We conclude that grain motion is mainly governed by the movement of the vices and by the upwards expansion of the sample. The coarse-grained sample shows more individuality in grain motions such that even an asymmetry with respect to the behavior of the left- and right-hand side builds up. In contrast, the grain motion of the fine-grained sample is more randomized and does not show this asymmetry.

\section{E. Grain rotation}

In the course of pressing, grains are not only translated, but also rotated. We calculate the rotation angle by using a quaternion formulation to identify rotation, which transforms the original orientation of each grain to the final orientation after pressing [46-48].

Fig. 8 shows the distribution of the rotation angles during the pressing phase. Quite large angles up to $8.5^{\circ}$ are observed with an average value of $1.29^{\circ}\left(1.40^{\circ}\right)$ for the coarse-grained (fine-grained) sample. The angles are approximately comparable for the 2 samples; however, the largest angles occur for the fine-grained sample. The effect of the grain rotation is the tilting of the grains at the lower sample surface visible in Fig. 4 and Fig. 5 which leads to the sample roughness discussed in Sect. III C. During the holding and release phase, rotation angles were $<0.01^{\circ}$, indicating that grains do not rotate back towards their initial orientations. This demonstrates that sizable rotations only occur under immediate sample loading.

\section{F. Sample distortion}

The length changes of the sample after release from the fixture are called distortions. We discuss here these length changes in all phases during and after the loading process. We measure these changes in vertical direction at 3 positions $\mathrm{A}$, $\mathrm{B}, \mathrm{C}$ at the left, middle and right of the sample, and at 3 positions in horizontal direction (D, E, F) in the bottom, middle, and top, see Fig. 9a. In detail, due to the atomistic roughness of our samples, for the example of position A, we measure the average height of the sample in a slab of width $1 \mathrm{~nm}$ extending at a distance of $3 \mathrm{~nm}$ from the lateral surface in the $z-y$ plane; this averaging process is performed because of the roughness of our samples due to their nano-crystalline structure, cf. Sect. III C.

Fig. 9 shows the distortions measured as fractional changes of the lengths with respect to their initial values. The sample shrank horizontally under the compressive loading by the vice plates. These induce a compressive strain of $-5 \%$ which exactly equals the distortion measured at $\mathrm{D}$ in the pressing and holding phase. After release, the sample does not return to its original length, since the deformation was plastic; the final distortion amounts to $-3.8(-2.7) \%$ for the coarse-grained (fine-grained) sample. Horizontal distortions in the middle of the sample at position $\mathrm{E}$ are considerably reduced, and at the sample top, position $\mathrm{F}$, the sample expands in all phases. This expansion is caused by material flow from the lower half of the sample to the upper half.

The horizontal narrowing of the sample is accompanied by an expansion in vertical direction. This expansion is more or less uniform for the fine-grained sample, but shows a considerable left-right asymmetry for the coarse-grained sample, which was already observed and discussed previously, Sect. III D.

We conclude that sample distortions after release are stronger for the coarse-grained than for the fine-grained sample. This is caused by the easier grain movement of the large 
grains, as evidenced in Sect. III D.

\section{SUMMARY}

We monitored the plastic deformation processes occurring in a polycrystalline $\mathrm{Al}$ bar under external inhomogeneous loading, representing vice plates. The processes occurring in the pressing, the holding and the release phases were analyzed and gave the following results.

1. Fine-grained material (grain size of $10 \mathrm{~nm}$ ) showed only little dislocation activity inside the grains during plastic deformation, while dislocation activity dominates the deformation of coarse-grained material (grain size of $18 \mathrm{~nm}$ ). This result is in agreement with previous studies of the deformation of nano-crystalline fcc metals $[12,13,16,42,44]$.

2. We followed the grain motion (sliding) by monitoring their center-of-mass trajectories. The results show strong deviations from a simple affine compaction of the material; rather, grains move in a strongly inhomogeneous way.

3. As a consequence of the nanometric size of the investigated Al bar, grain motions are not symmetric with respect to the bar center, but exhibit a left-right asymmetry. It is caused by grain sliding and rotation and occurs pronouncedly in the coarse-grained sample, while it averages out in the fine-grained sample. It may be expected that this asymmetry will decrease for larger samples.

4. The grains also suffer considerable rotations with an average rotation angle of $1.3^{\circ}-1.4^{\circ}$. These grain rotations only occurred in the pressing phase but not during holding or releasing.

5. These grain rotations lead to a considerable roughening of the bottom surface of the sample, which does not vanish after releasing the vice fixtures.

6. The residual stresses in the material resulting from dislocation activity and grain-boundary processes build up in the pressing phase and do not relax during the holding and release phases.

7. Due to the plastic deformations occurring, the sample shows a distortion after the process. The final distortion at the position where the vice plates acted amounts to around half the applied strain, but decreases in other areas. In the direction perpendicular to the applied strain, the sample grows. The coarse-grained sample shows higher distortion values than the fine-grained sample because of longer-ranged grain translation.

Our results show that the final sample distortion is based on plastic processes that are mediated both by dislocation glide and grain-boundary sliding and rotation. In future work, the results presented here should be extended to other material classes. Besides bcc metals, such as Fe, the distortion processes in alloys would be particularly relevant.

\section{DECLARATIONS}

\section{Funding}

We acknowledge support by the Deutsche Forschungsgemeinschaft (DFG, German Research Foundation) - project number 252408385 - IRTG 2057.

\section{Conflict of interest}

The authors declare that they have no conlict of interest.

\section{Availability of data and material}

This study is based only on data obtained using the methods described in this paper.

\section{Code availability}

The simulations were performed by the open-source code LAMMPS available under http://lammps.sandia.gov/ [34].

\section{ACKNOWLEDGMENTS}

Simulations were performed at the High Performance Cluster Elwetritsch (RHRK, TU Kaiserslautern, Germany).
[1] George Ellwood Dieter, Mechanical metallurgy, 3rd ed. (McGraw-Hill, 1986).

[2] Daniel Weber, Benjamin Kirsch, Christopher R. D'Elia, Barbara S. Linke, Michael R. Hill, and Jan C. Aurich, "Concept to analyze residual stresses in milled thin walled monolithic aluminum components and their effect on part distortion," in Production at the leading edge of technology, edited by Jens Pe- ter Wulfsberg, Wolfgang Hintze, and Bernd-Arno Behrens (Springer, Berlin, Heidelberg, 2019) pp. 287-296.

[3] Fuping Yuan and Xiaolei Wu, "Size effects of primary/secondary twins on the atomistic deformation mechanisms in hierarchically nanotwinned metals," Journal of Applied Physics 113, 203516 (2013). 
[4] L. DAlvise, D. Chantzis, B. Schoinochoritis, and K. Salonitis, "Modelling of part distortion due to residual stresses relaxation: An aeronautical case study," Procedia CIRP 31, 447-452 (2015), 15th CIRP Conference on Modelling of Machining Operations (15th CMMO).

[5] Xing Ke, Jianchao Ye, Zhiliang Pan, Jie Geng, Matt F. Besser, Dongxia Qu, Alfredo Caro, Jaime Marian, Ryan T. Ott, Y. Morris Wang, and Frederic Sansoz, "Ideal maximum strengths and defect-induced softening in nanocrystalline-nanotwinned metals," Nature Materials 18, 1207-1214 (2019).

[6] M. Aurrekoetxea, L. N. López de Lacalle, and I. Llanos, "Machining stresses and initial geometry on bulk residual stresses characterization by on-machine layer removal," Materials 13, 1445 (2020).

[7] Zhiqiang Fan, Lixin Cao, and Feng Liu, "FEM analysis of the distortion of thin-walled sealing part affected by the machininginduced residual stress," IOP Conference Series: Materials Science and Engineering 768, 042028 (2020).

[8] Jan C. Aurich, Marina Carrella, and Michael Walk, "Micro grinding with ultra small micro pencil grinding tools using an integrated machine tool," CIRP Ann. - Manuf. Technol. 64, 325 -328 (2015).

[9] Bharat Bhushan, ed., Springer Handbook of Nanotechnology, 4th ed. (Springer, Heidelberg, 2017).

[10] S. B. Sinnott, S.-J. Heo, D. W. Brenner, J. A. Harrison, and D. L. Irving, "Computer simulations of nanometer-scale indentation and friction," in Springer Handbook of Nanotechnology, edited by Bharat Bhushan (Springer, Heidelberg, 2010) 3rd ed.

[11] William Gerberich, Ellad B. Tadmor, Jeffrey Kysar, Jonathan A. Zimmerman, Andrew M. Minor, Izabela Szlufarska, Jonathan Amodeo, Benoit Devincre, Eric Hintsala, and Roberto Ballarini, "Review article: Case studies in future trends of computational and experimental nanomechanics," Journal of Vacuum Science \& Technology A: Vacuum, Surfaces, and Films 35, 060801 (2017).

[12] Jakob Schiøtz, Francesco D. Di Tolla, and Karsten W. Jacobsen, "Softening of nanocrystalline metals at very small grain sizes," Nature 391, 561-563 (1998).

[13] Helena Van Swygenhoven and Julia R. Weertman, "Deformation in nanocrystalline metals," Materials Today 9, 24-31 (2006).

[14] T. Shimokawa, A. Nakatani, and H. Kitagawa, "Grain-size dependence of the relationship between intergranular and intragranular deformation of nanocrystalline al by molecular dynamics simulations," Phys. Rev. B 71, 224110 (2005).

[15] H. Huang and H. Van Swygenhoven, "Atomistic simulations of mechanics of nanostructures," MRS Bull. 34, 160 (2009).

[16] Xiaoyan Li, Yujie Wei, Wei Yang, and Huajian Gao, "Competing grain-boundary- and dislocation-mediated mechanisms in plastic strain recovery in nanocrystalline aluminum," Proceedings of the National Academy of Sciences 106, 16108-16113 (2009).

[17] Luis A. Zepeda-Ruiz, Alexander Stukowski, Tomas Oppelstrup, and Vasily V. Bulatov, "Probing the limits of metal plasticity with molecular dynamics simulations," Nature 550, 492 (2017).

[18] Kai Zhou, Bin Liu, Shaofeng Shao, and Yijun Yao, "Molecular dynamics simulations of tensioncompression asymmetry in nanocrystalline copper," Physics Letters A 381, 1163-1168 (2017).

[19] Xiaoling Zhou, Zongqiang Feng, Linli Zhu, Jianing Xu, Lowell Miyagi, Hongliang Dong, Hongwei Sheng, Yanju Wang, Quan Li, Yanming Ma, Hengzhong Zhang, Jinyuan Yan, Nobumichi Tamura, Martin Kunz, Katie Lutker, Tianlin Huang,
Darcy A. Hughes, Xiaoxu Huang, and Bin Chen, "Highpressure strengthening in ultrafine-grained metals," Nature 579, 67-72 (2020).

[20] C. C. Huang, T. C. Chiang, and T. H. Fang, "Grain size effect on indentation of nanocrystalline copper," Appl. Surf. Sci. 353, 494 - 498 (2015).

[21] Carlos J Ruestes, Eduardo M Bringa, Yu Gao, and Herbert M Urbassek, "Molecular dynamics modeling of nanoindentation," in Applied Nanoindentation in Advanced Materials, edited by Atul Tiwari and Sridhar Natarajan (Wiley, Chichester, UK, 2017) Chap. 14, pp. 313-345.

[22] Iyad Alabd Alhafez, Alexander Brodyanski, Michael Kopnarski, and Herbert M. Urbassek, "Influence of tip geometry on nanoscratching," Tribol. Lett. 65, 26 (2017).

[23] Yu Gao and Herbert M. Urbassek, "Evolution of plasticity in nanometric cutting of Fe single crystals," Appl. Surf. Sci. 317, 6 - 10 (2014).

[24] M. A. Meyers and Krishan K. Chawla, Mechanical behavior of materials, 2nd ed. (Cambridge University Press, Cambridge, 2010).

[25] Diana Farkas, Som Mohanty, and Joshua Monk, "Strain-driven grain boundary motion in nanocrystalline materials," Materials Science and Engineering: A 493, 33-40 (2008), mechanical Behavior of Nanostructured Materials, a Symposium Held in Honor of Carl Koch at the TMS Annual Meeting 2007, Orlando, Florida.

[26] N. Q. Vo, R. S. Averback, P. Bellon, S. Odunuga, and A. Caro, "Quantitative description of plastic deformation in nanocrystalline cu: Dislocation glide versus grain boundary sliding," Phys. Rev. B 77, 134108 (2008).

[27] N. Zaafarani, D. Raabe, F. Roters, and S. Zaefferer, "On the origin of deformation-induced rotation patterns below nanoindents," Acta Mater. 56, 31 (2008).

[28] Pedro A. Romero, Tommi T. Järvi, Nils Beckmann, Matous Mrovec, and Michael Moseler, "Coarse graining and localized plasticity between sliding nanocrystalline metals," Phys. Rev. Lett. 113, 036101 (2014).

[29] Destiny R. Garcia, Zhibo Zhang, Barbara S. Linke, and Herbert M. Urbassek, "Molecular dynamics simulations of single grain pure aluminum in a vice fixture for nanomanufacturing applications," CIRP J. Manuf. Sci. Technol. 23, 91 - 97 (2018).

[30] G. Voronoi, "Nouvelles applications des paramètres continus à la théorie des formes quadratiques," Reine Angew. Math. 134, 199 (1908)

[31] Pierre Hirel, "Atomsk: A tool for manipulating and converting atomic data files," Comput. Phys. Commun. 197, 212-219 (2015).

[32] A. Prakash, M. Hummel, S. Schmauder, and E. Bitzek, "Nanosculpt: A methodology for generating complex realistic configurations for atomistic simulations," MethodsX 3, 219 230 (2016).

[33] M. I. Mendelev, M. J. Kramer, C. A. Becker, and M. Asta, "Analysis of semi-empirical interatomic potentials appropriate for simulation of crystalline and liquid $\mathrm{Al}$ and $\mathrm{Cu}$," Philos. Mag. 88, 1723-1750 (2008).

[34] St. Plimpton, "Fast parallel algorithms for short-range molecular dynamics," J. Comput. Phys. 117, 1-19 (1995), http://lammps.sandia.gov/.

[35] A. Stukowski, "Visualization and analysis of atomistic simulation data with OVITO - the Open Visualization Tool," Model. Simul. Mater. Sci. Eng. 18, 015012 (2010), http://www.ovito.org/.

[36] Zhibo Zhang and Herbert M. Urbassek, "Indentation into an Al-Si composite: enhanced dislocation mobility at interface," J. 
Mater. Sci. 53, 799-813 (2018).

[37] E. Bitzek, P. M. Derlet, P. M. Anderson, and H. Van Swygenhoven, "The stress-strain response of nanocrystalline metals: A statistical analysis of atomistic simulations," Acta Materialia 56, 4846 - 4857 (2008).

[38] V. Dupont and F. Sansoz, "Quasicontinuum study of incipient plasticity under nanoscale contact in nanocrystalline aluminum," Acta Materialia 56, 6013-6026 (2008).

[39] K. Kadau, T. C. Germann, P. S. Lomdahl, B. L. Holian, D. Kadau, P. Entel, M. Kreth, F. Westerhoff, and D. E. Wolf, "Molecular-dynamics study of mechanical deformation in nano-crystalline aluminum," Metallurgical and Materials Transactions A 35, 2719 (2004).

[40] A. Prakash, D. Weygand, and E. Bitzek, "Influence of grain boundary structure and topology on the plastic deformation of nanocrystalline aluminum as studied by atomistic simulations," International Journal of Plasticity 97, 107 - 125 (2017).

[41] Avik Mahata and Mohsen Asle Zaeem, "Evolution of solidification defects in deformation of nano-polycrystalline aluminum," Computational Materials Science 163, 176-185 (2019).

[42] Jakob Schiøtz, "Atomic-scale modeling of plastic deformation of nanocrystalline copper," Scripta Materialia 51, 837-841 (2004), viewpoint set no. 35. Metals and alloys with a structural scale from the micrometer to the atomic dimensions.

[43] J. Schiøtz, T. Vegge, F. D. Di Tolla, and K. W. Jacobsen, "Atomic-scale simulations of the mechanical deformation of nanocrystalline metals," Phys. Rev. B 60, 11971 (1999).

[44] Jakob Schiøtz and Karsten W. Jacobsen, "A maximum in the strength of nanocrystalline copper," Science 301, 1357 - 1359 (2003).

[45] Ankit Gupta, Jacob Gruber, Satish S. Rajaram, Gregory B. Thompson, David L. McDowell, and Garritt J. Tucker, "On the mechanistic origins of maximum strength in nanocrystalline metals," npj Computational Materials 6, 153 (2020).

[46] Luis Antonio Barrales Mora, 2D and 3D Grain Growth Modeling and Simulation, Ph.D. thesis, RWTH Aachen (2008).

[47] Samir El Shawish and Leon Cizelj, "Numerical investigation of grain misorientations at and close to the free surface of fcc polycrystalline metals," Computational Materials Science 113, 133-142 (2016).

[48] Matthew Kasemer, Eloisa Zepeda-Alarcon, Robert Carson, Paul Dawson, and Hans-Rudolf Wenk, "Deformation heterogeneity and intragrain lattice misorientation in high strength contrast, dual-phase bridgmanite/periclase," Acta Materialia 189, 284-298 (2020). 
TABLE 1. Average (avg) and standard deviation (std) of the surface profiles, Fig. 6. Data are in $\AA$ units.

\begin{tabular}{r|ll|ll|ll|ll}
\hline sample & \multicolumn{2}{|c|}{ initial } & \multicolumn{2}{c|}{ pressing } & \multicolumn{2}{c}{ holding } & \multicolumn{2}{c}{ release } \\
& avg & std & avg & std & level $\AA$ & std & avg & std \\
\hline coarse-grained & -304.42 & 0.60 & -305.85 & 4.15 & -305.30 & 7.12 & -306.61 & 5.94 \\
fine-grained & -305.05 & 0.60 & -305.13 & 2.62 & -303.97 & 3.21 & -303.31 & 2.63 \\
\hline
\end{tabular}

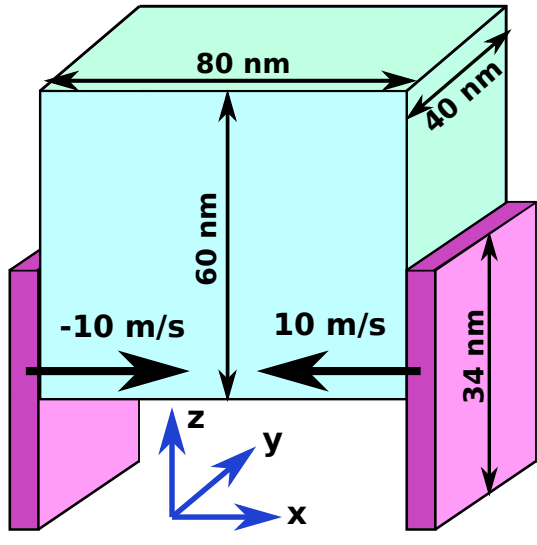

(a)

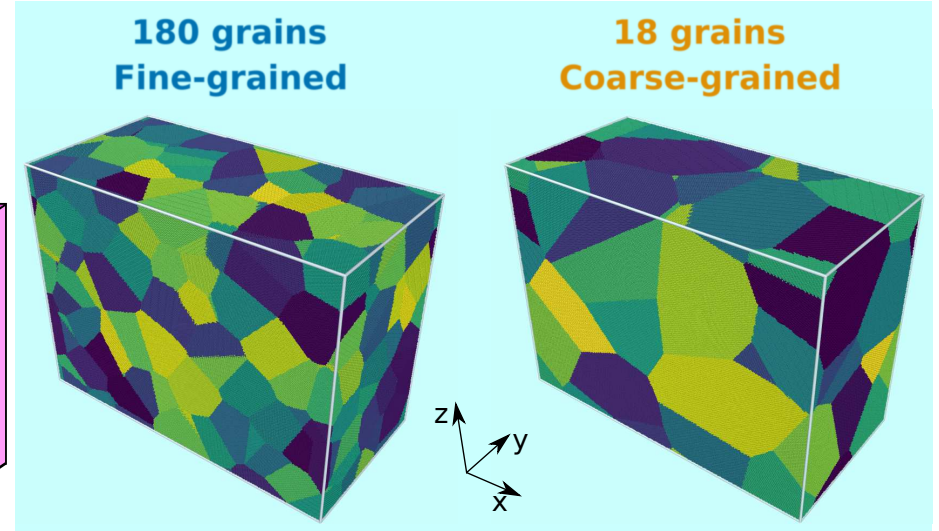

(b)

FIG. 1. a) Schematic setup of the simulation system. The vice plates compress the Al bar laterally. b) Two simulation samples containing 180 and 18 grains with average grains sizes of $10 \mathrm{~nm}$ and $22 \mathrm{~nm}$, respectively.

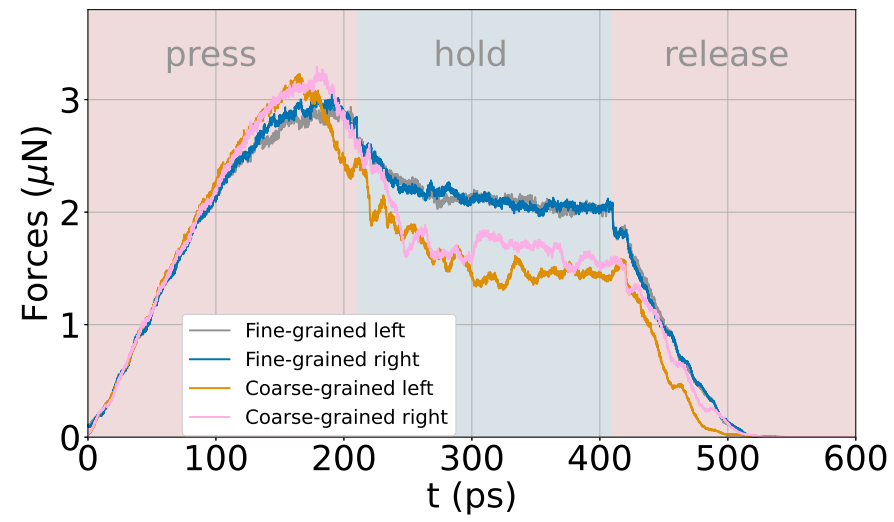

FIG. 2. Time evolution of the forces acting in $x$ direction on the vices. 


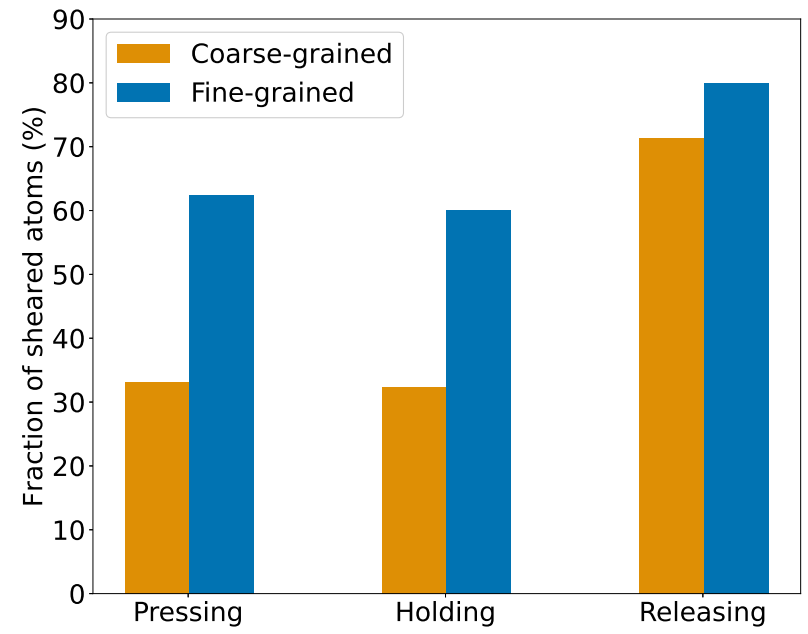

FIG. 3. Fraction of grain-boundary atoms with VMSS $>0.1$. The reference configurations for holding and releasing are the end of the pressing and the holding phase, respectively.

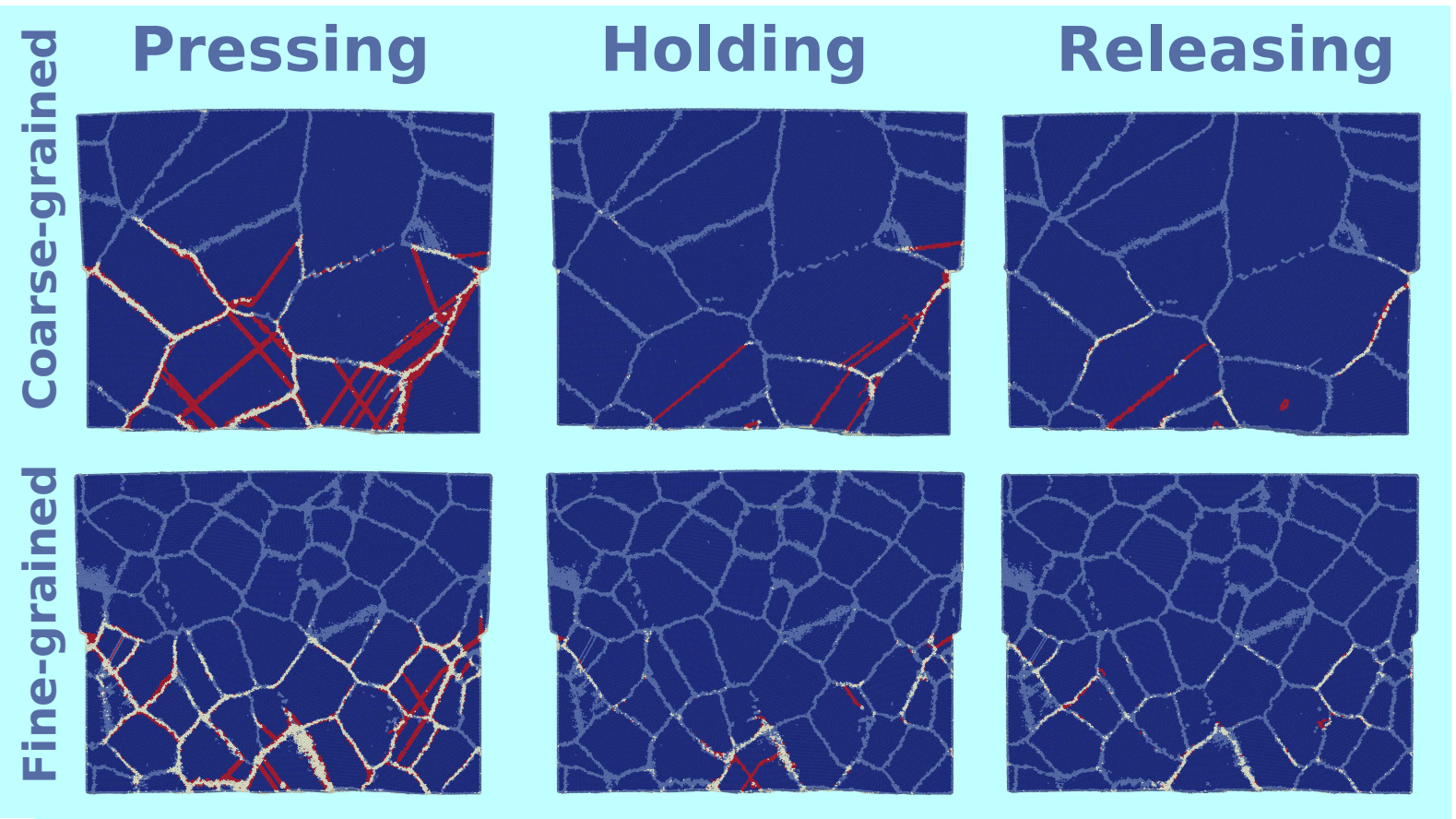

FIG. 4. VMSS map of coarse-grained and fine-grained samples, during pressing, holding and release. The reference configurations are the same as in Fig. 3. In this side view on the sample, dark blue represents atoms in fcc lattice structure inside the grains and light blue represents atoms at grain boundaries. White characterizes grain-boundary atoms with VMSS $>0.1$, and red atoms have VMSS $>0.1$ and belong to the grain interior. 


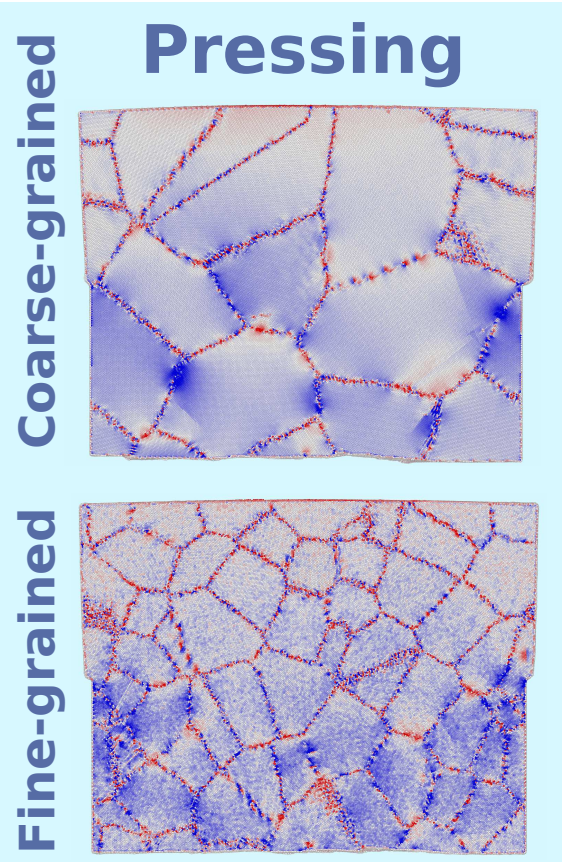

\section{Holding}
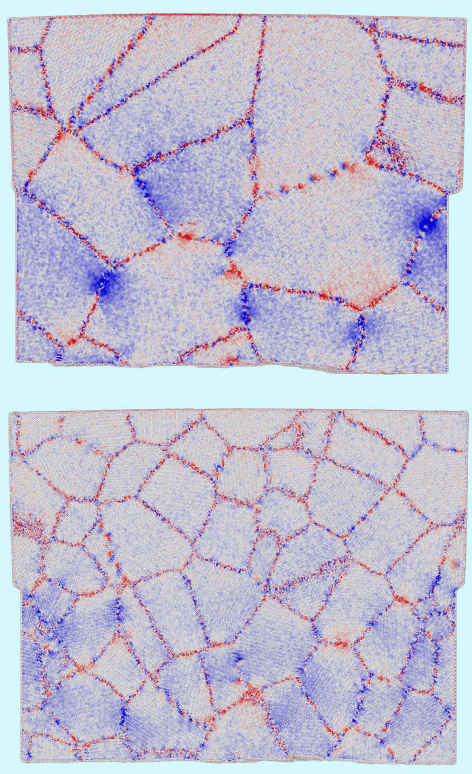

Hydrostatic pressure
Releasing
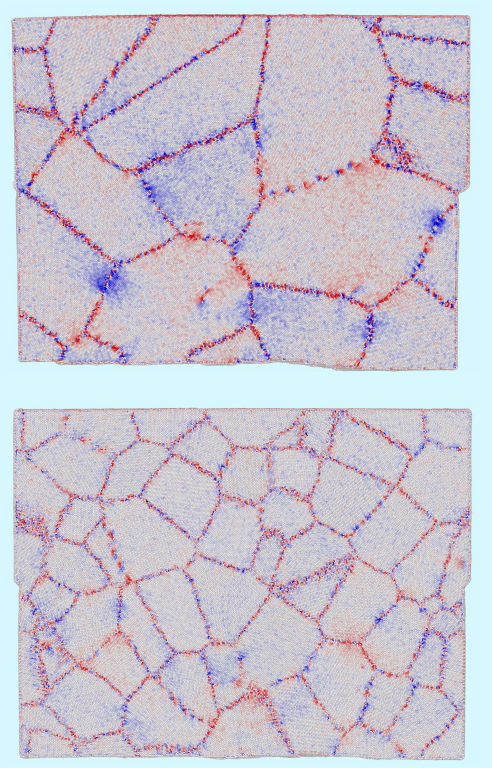
$-4 \mathrm{GPa}$

FIG. 5. Hydrostatic pressure. Negative values denote compressive pressure. 

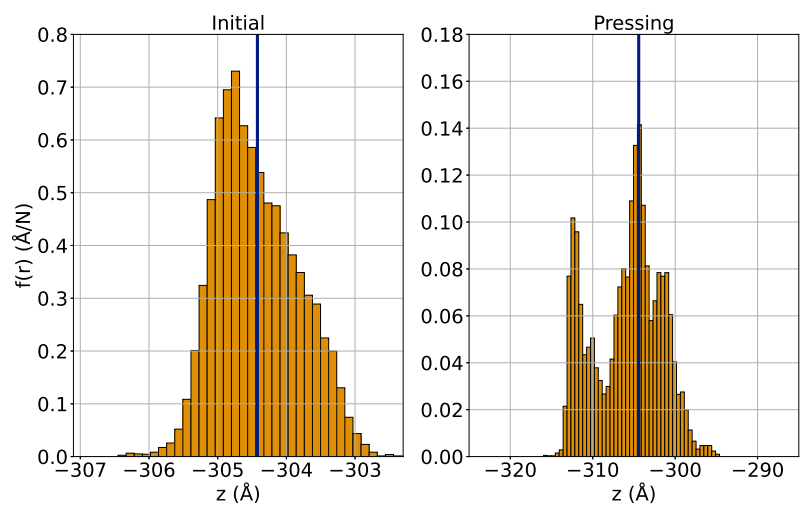

(a)
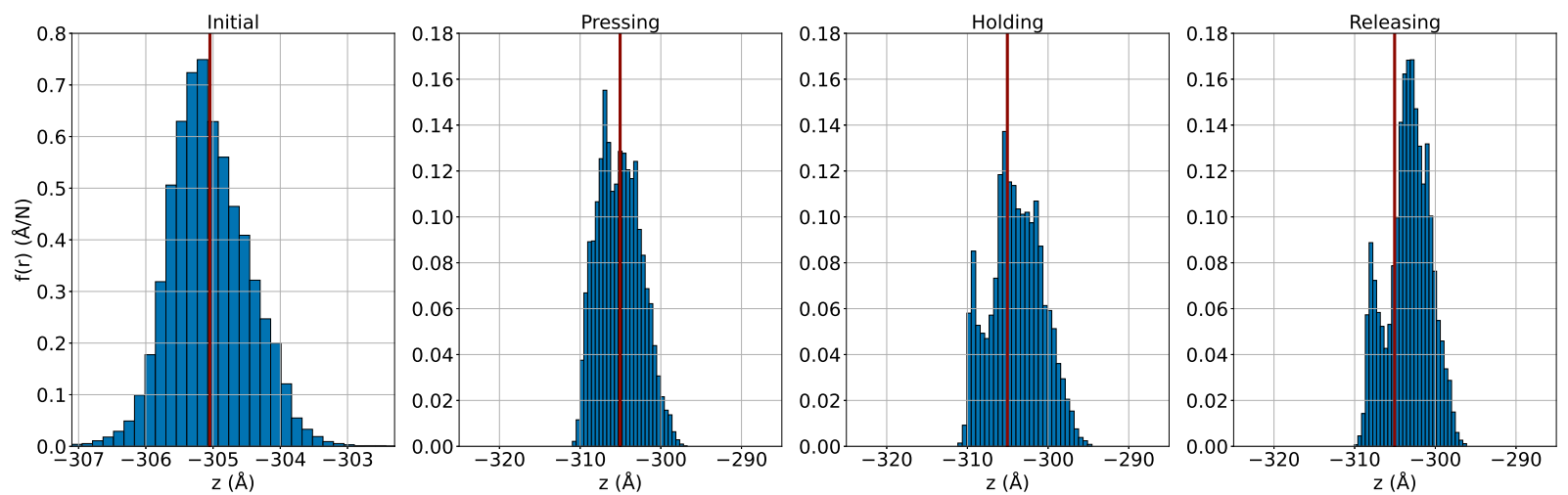

(b)

FIG. 6. Height profile of the bottom sample surface for the (a) coarse-grained sample and b) fine-grained sample. The vertical line indicates the original average surface position. Note the change in scale between the initial and the deformed profiles. 


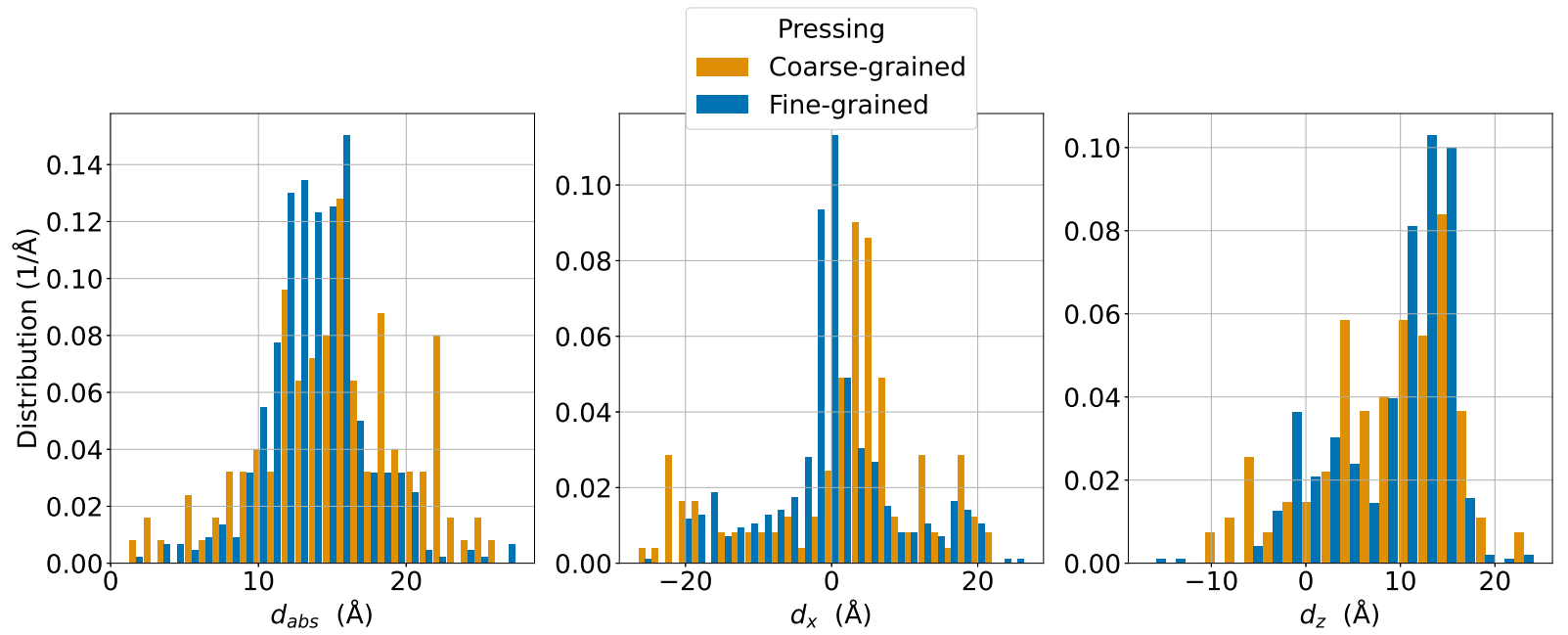

(a)
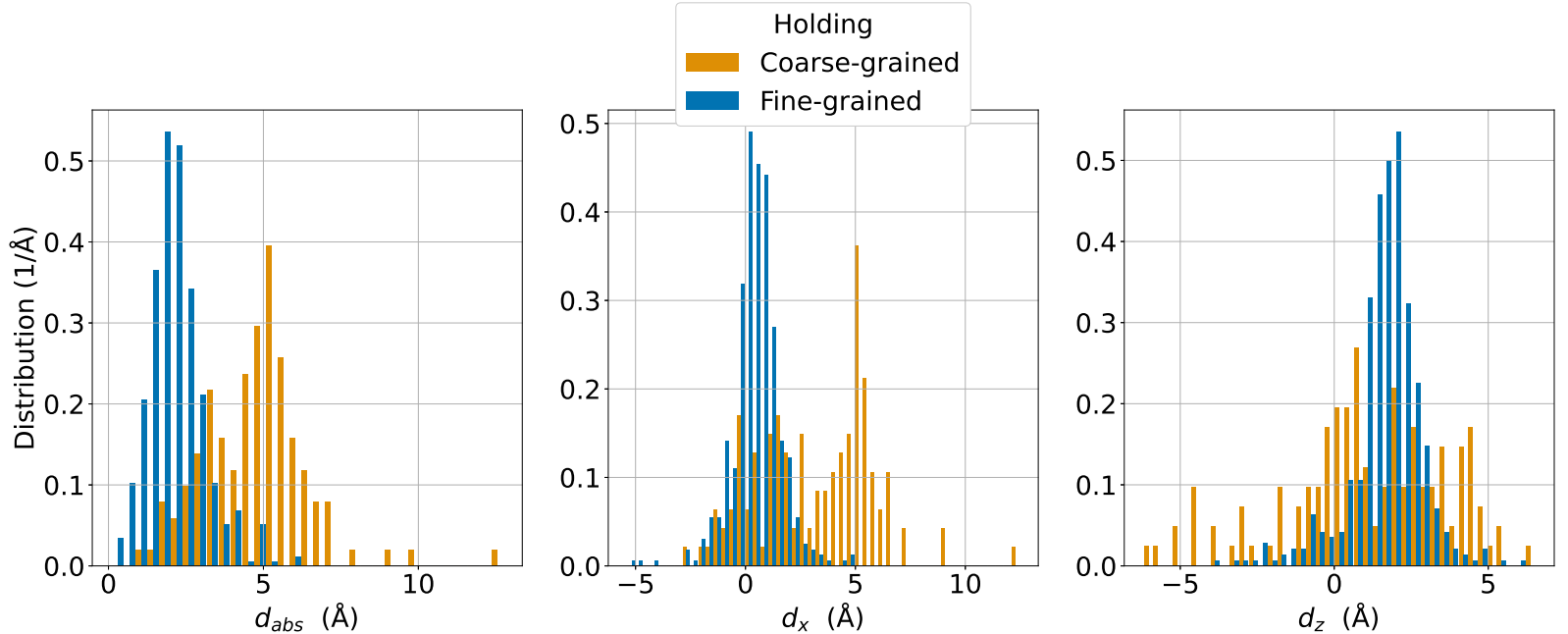

(b)

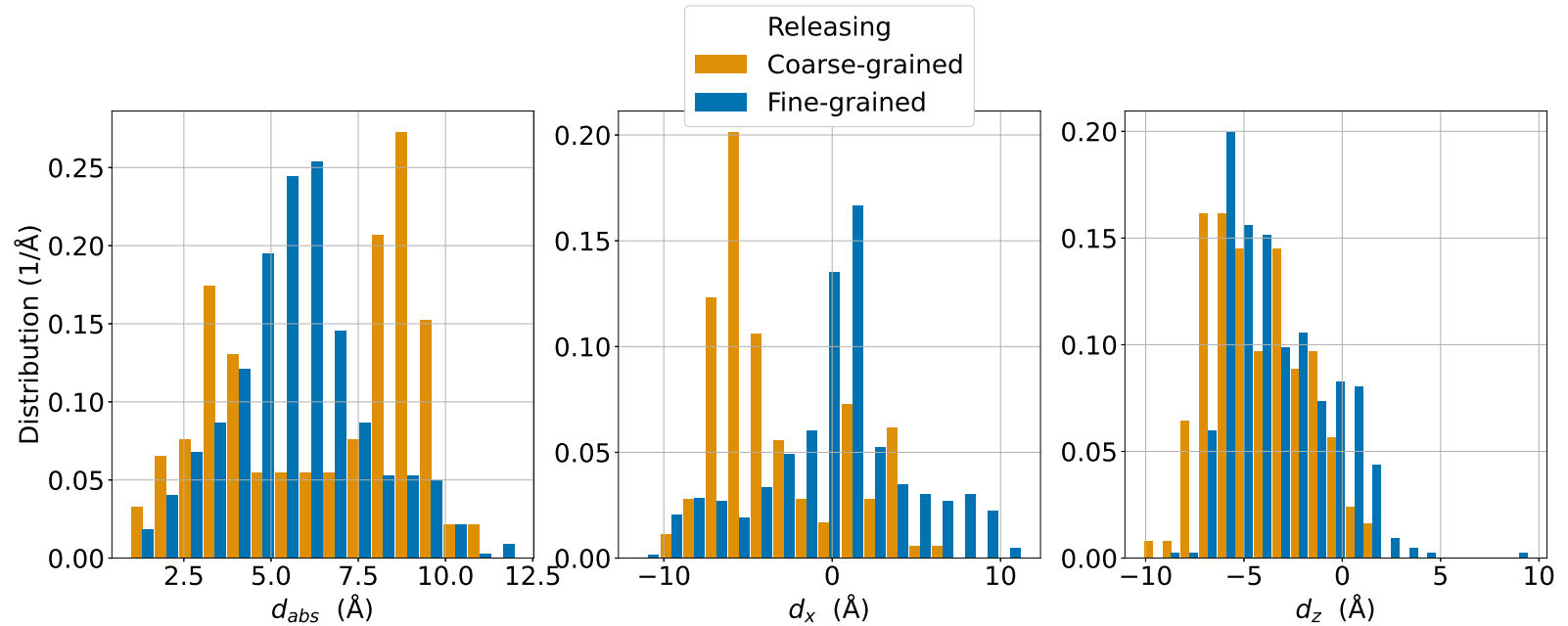

(c)

FIG. 7. Normalized distributions of the center-of-mass displacements of grains during the (a) pressing, (b) holding, and (c) release phase. 


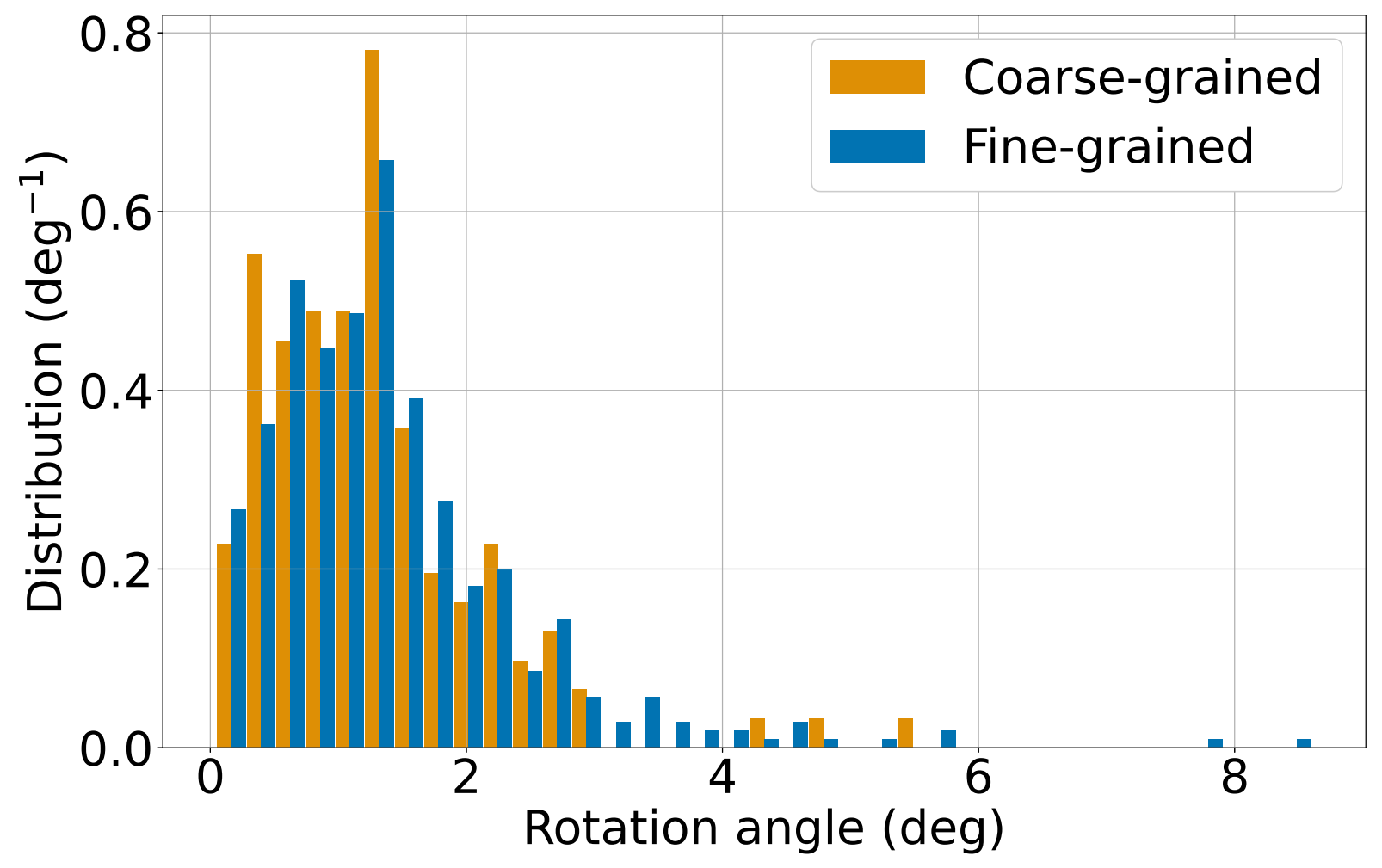

FIG. 8. Distribution of grain rotation angles during the pressing phase. 


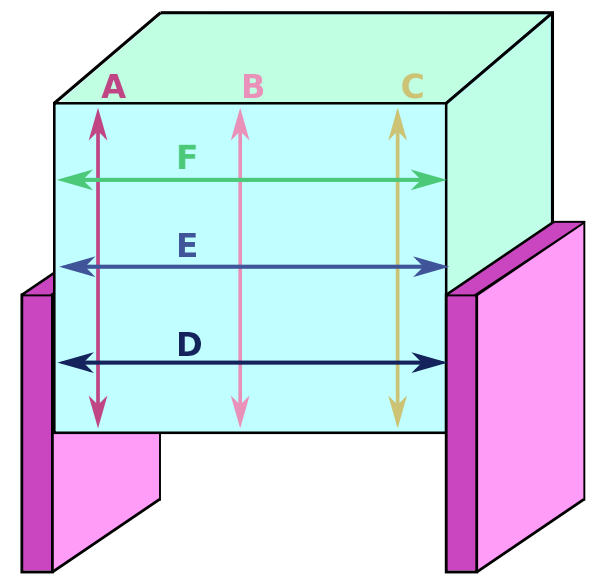

(a)

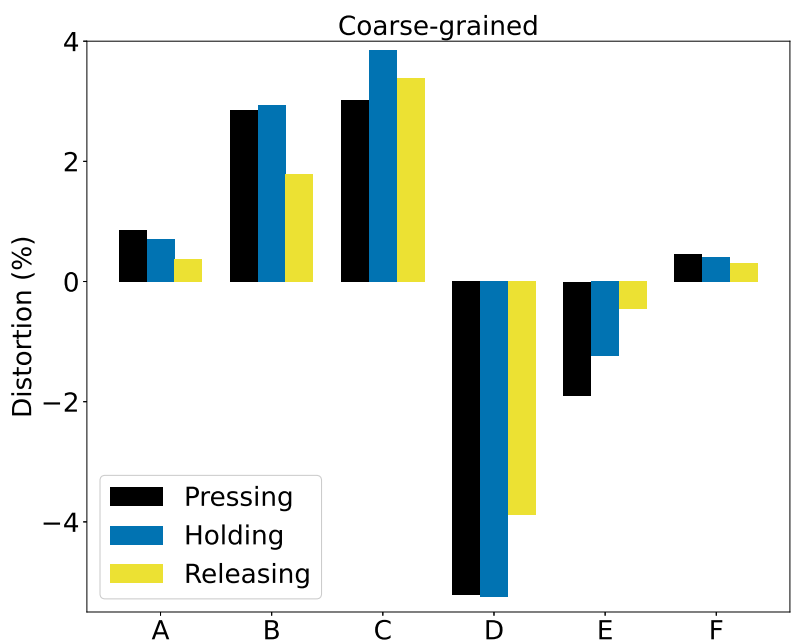

(b)

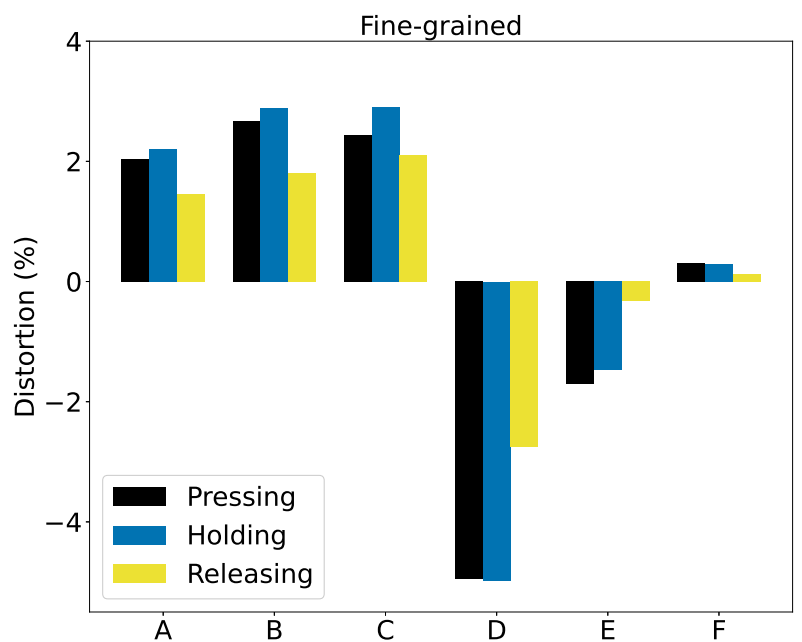

(c)

FIG. 9. (a) Schematics indicating the positions where vertical (A-C) and horizontal (F-D) sample distortions were measured. (b) and (c) show simulation results for distortions for the coarse-grained (b) and fine-grained (c) sample in the pressing, holding and release phase. 


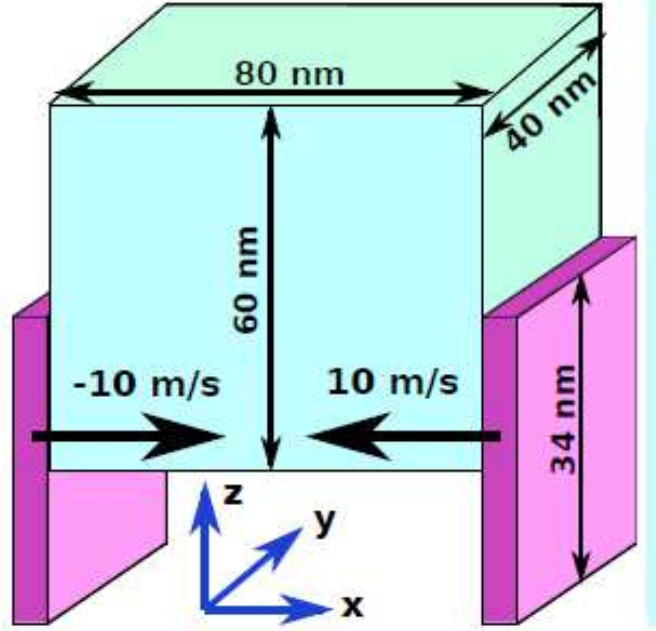

(a)

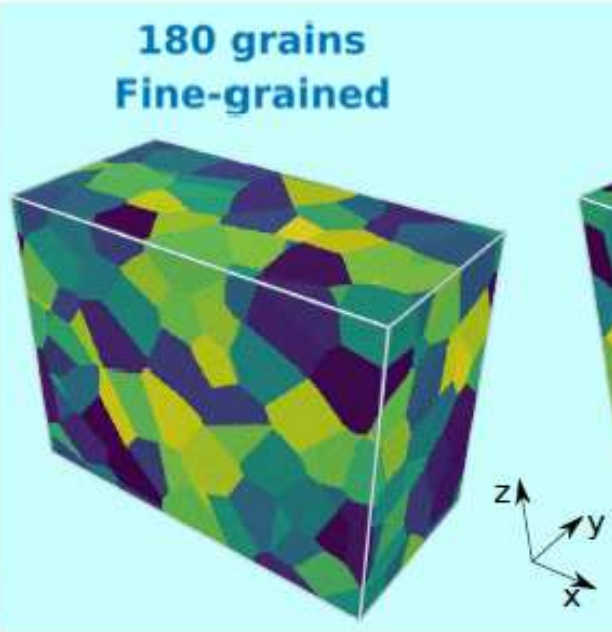

(b)

\section{Figure 1}

a) Schematic setup of the simulation system. The vice plates compress the Al bar laterally. b) Two simulation samples containing 180 and 18 grains with average grains sizes of $10 \mathrm{~nm}$ and $22 \mathrm{~nm}$, respectively.

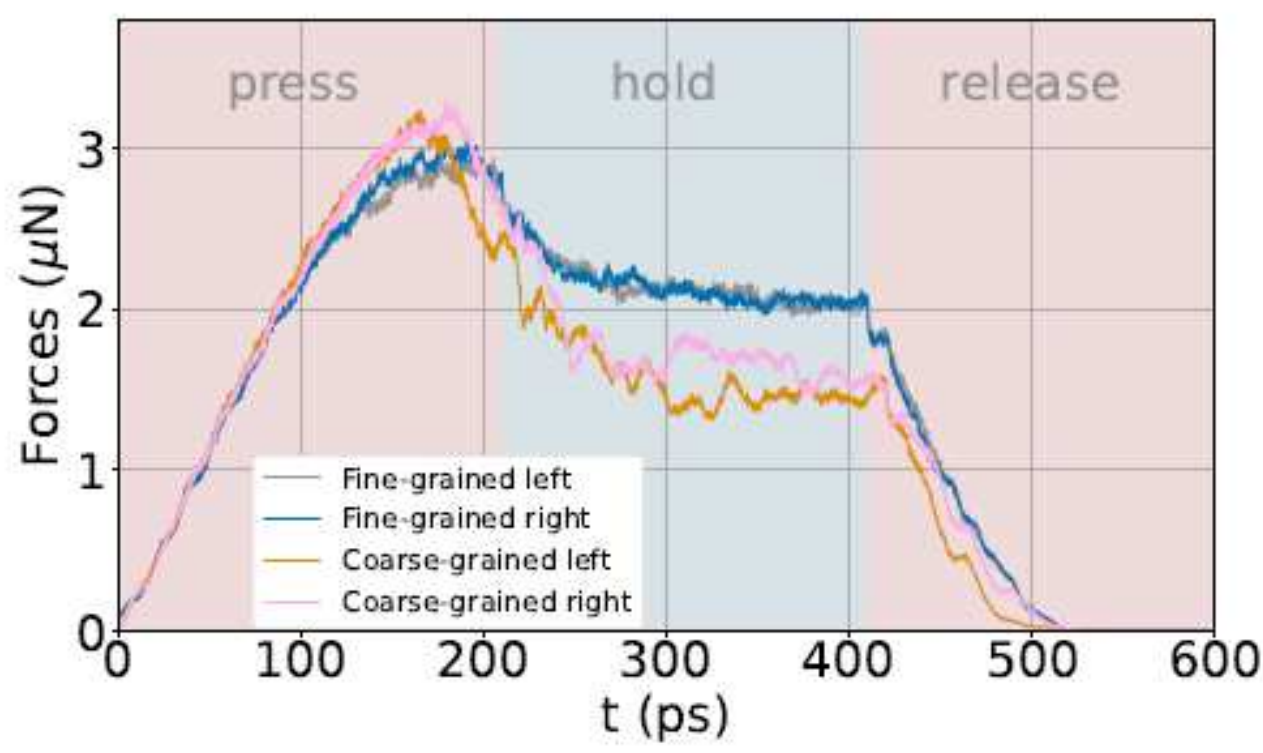

Figure 2

Time evolution of the forces acting in $\mathrm{x}$ direction on the vices. 


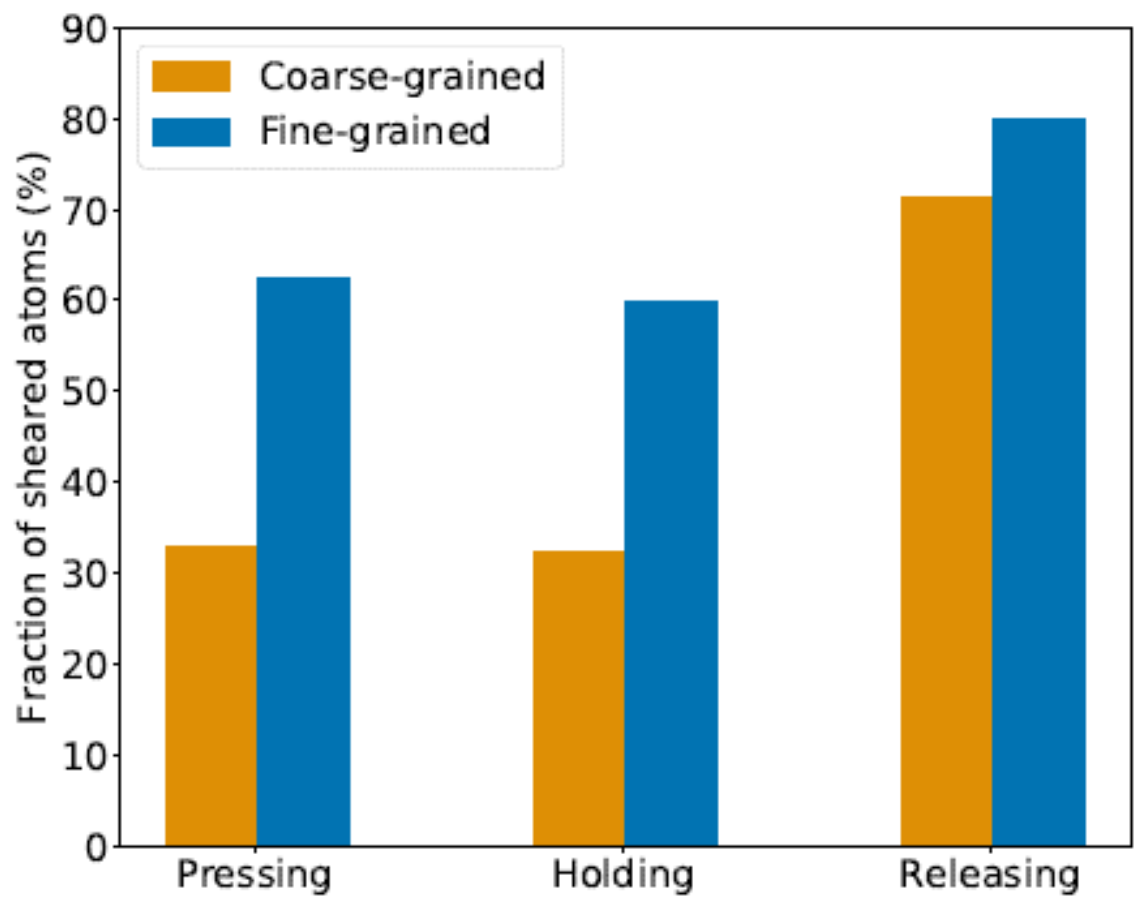

Figure 3

Fraction of grain-boundary atoms with VMSS $>0.1$. The reference configurations for holding and releasing are the end of the pressing and the holding phase, respectively.

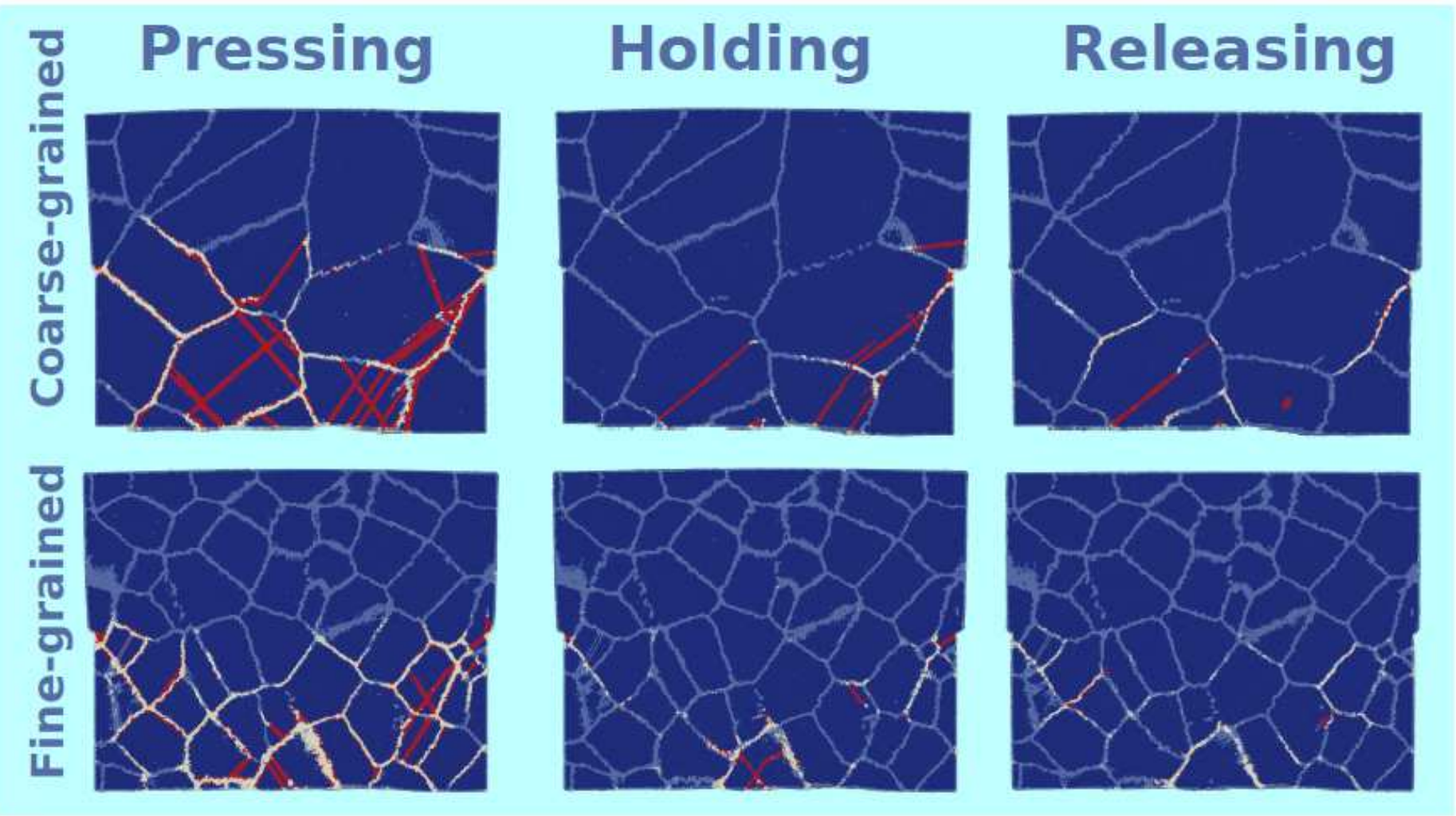


VMSS map of coarse-grained and fine-grained samples, during pressing, holding and release. The reference configurations are the same as in Fig. 3. In this side view on the sample, dark blue represents atoms in fcc lattice structure inside the grains and light blue represents atoms at grain boundaries. White characterizes grain-boundary atoms with VMSS $>0.1$, and red atoms have VMSS $>0.1$ and belong to the grain interior.

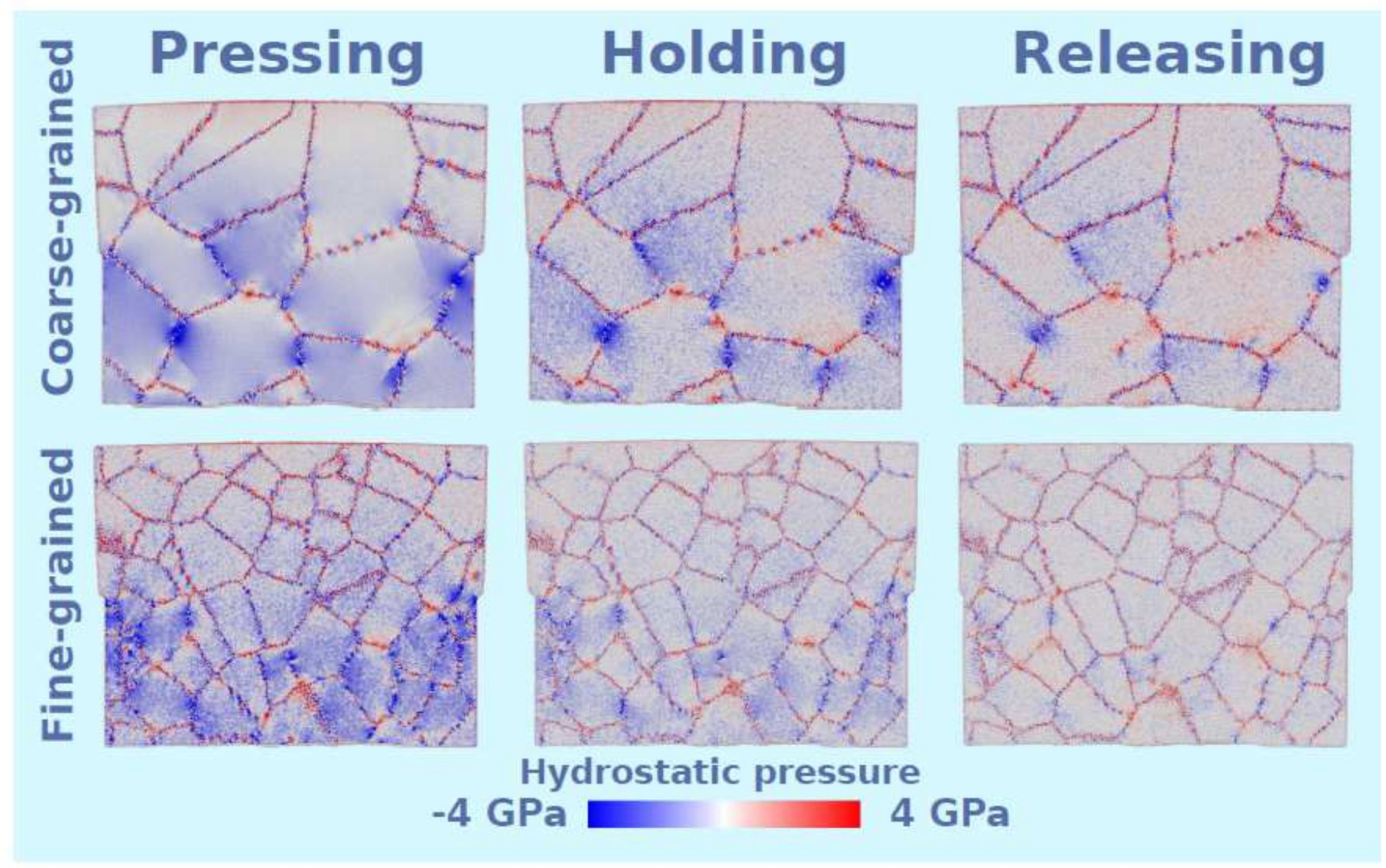

Figure 5

Hydrostatic pressure. Negative values denote compressive pressure. 

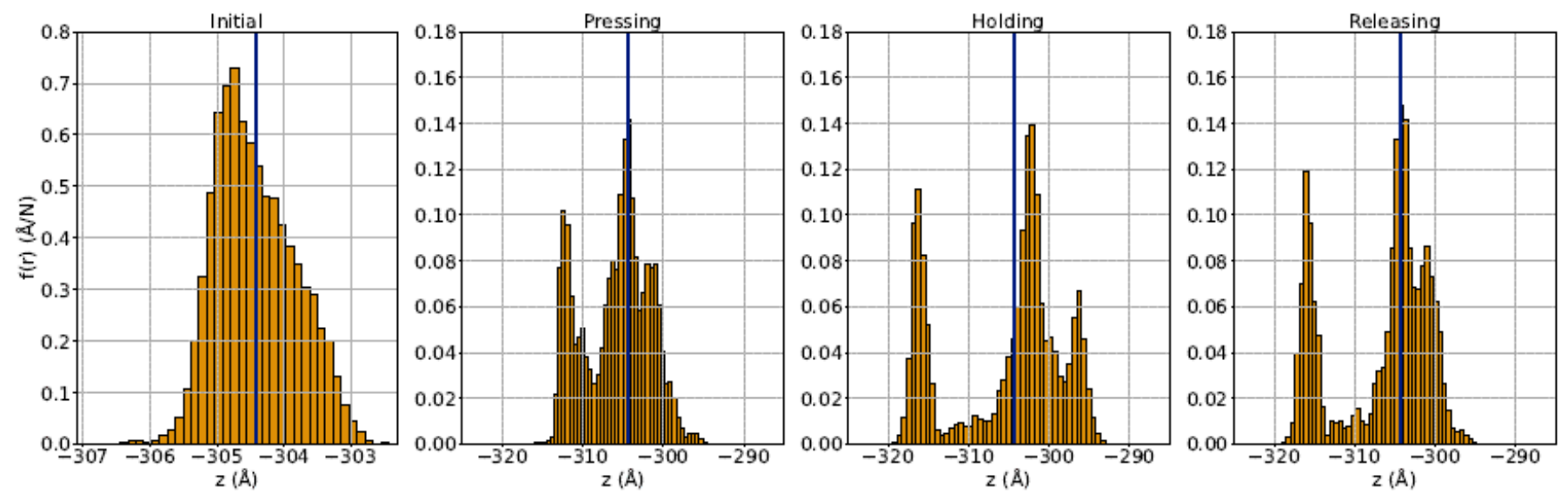

(a)
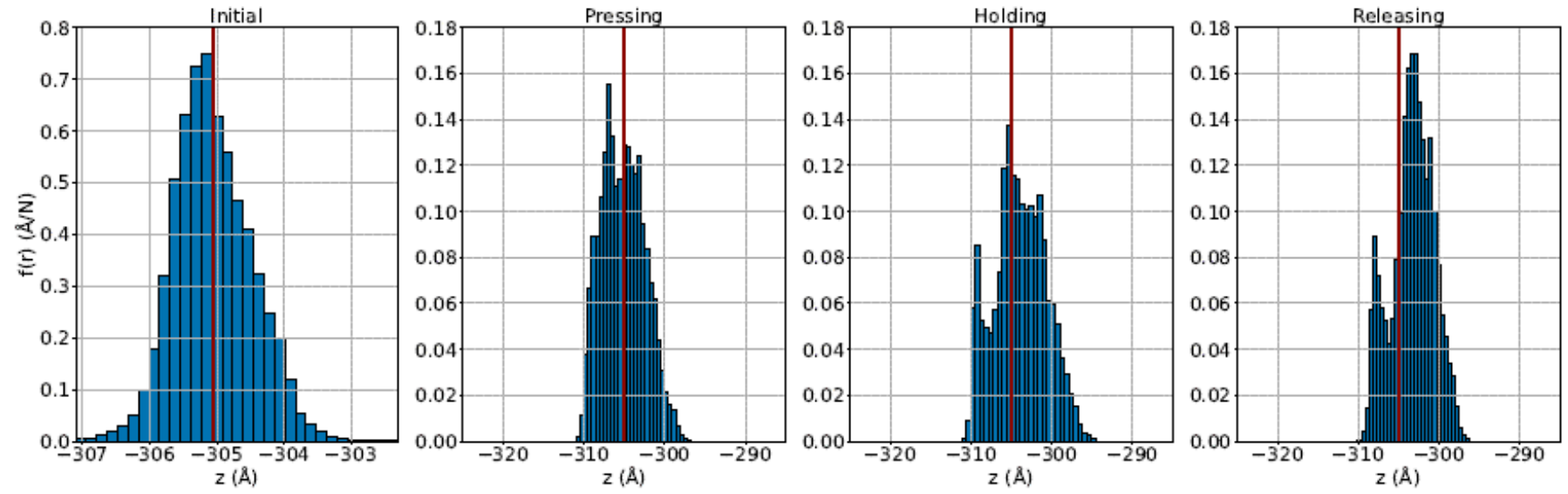

(b)

Figure 6

Height profile of the bottom sample surface for the (a) coarse-grained sample and b) fine-grained sample. The vertical line indicates the original average surface position. Note the change in scale between the initial and the deformed profiles. 


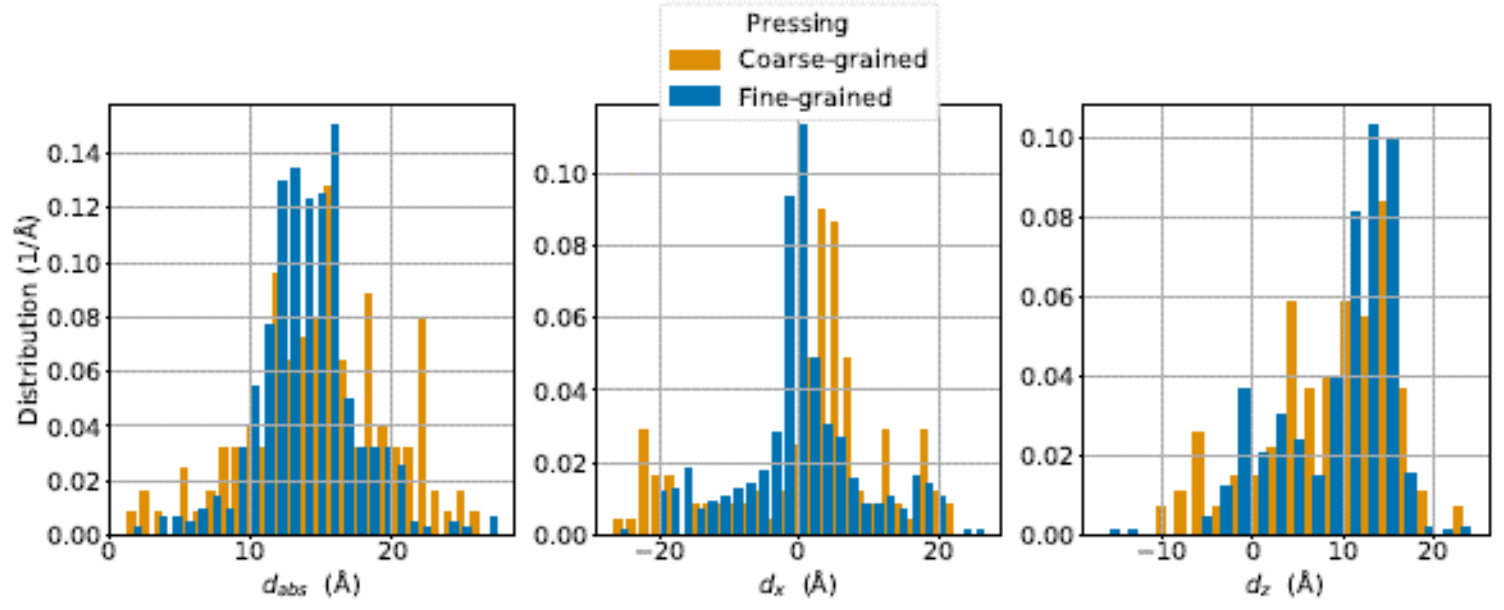

(a)
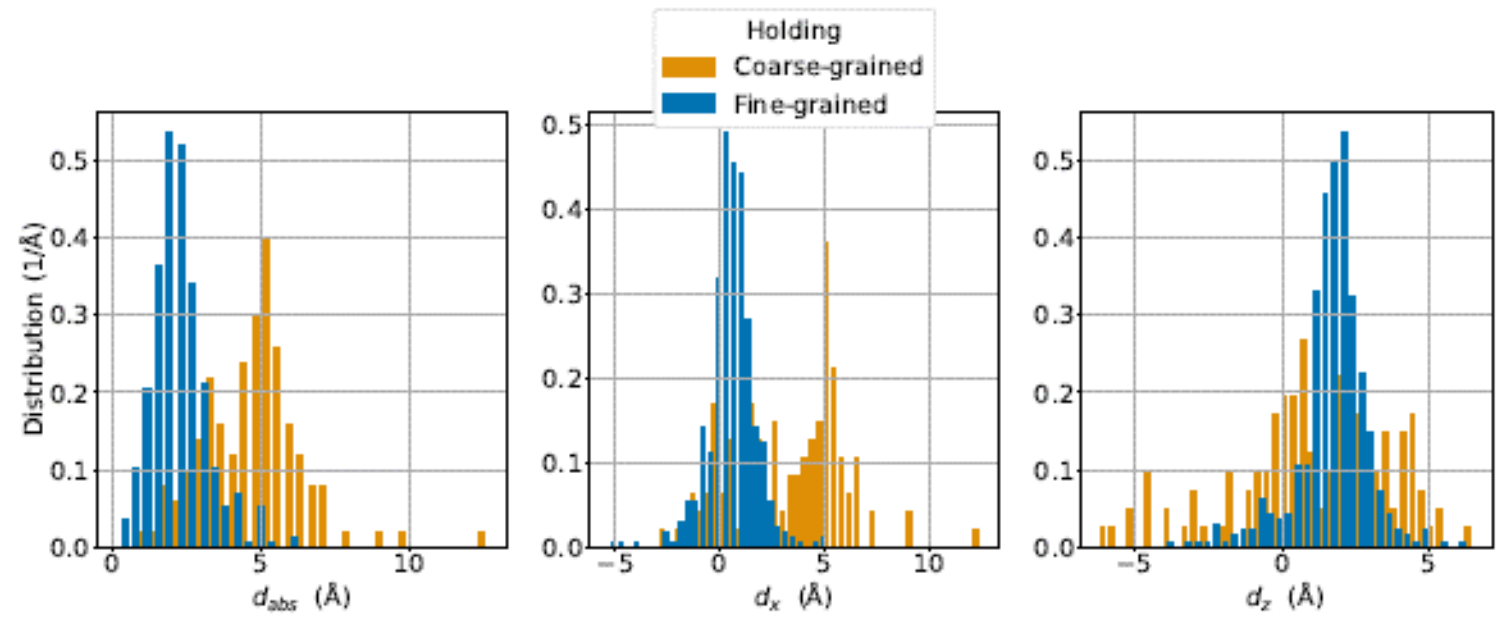

(b)
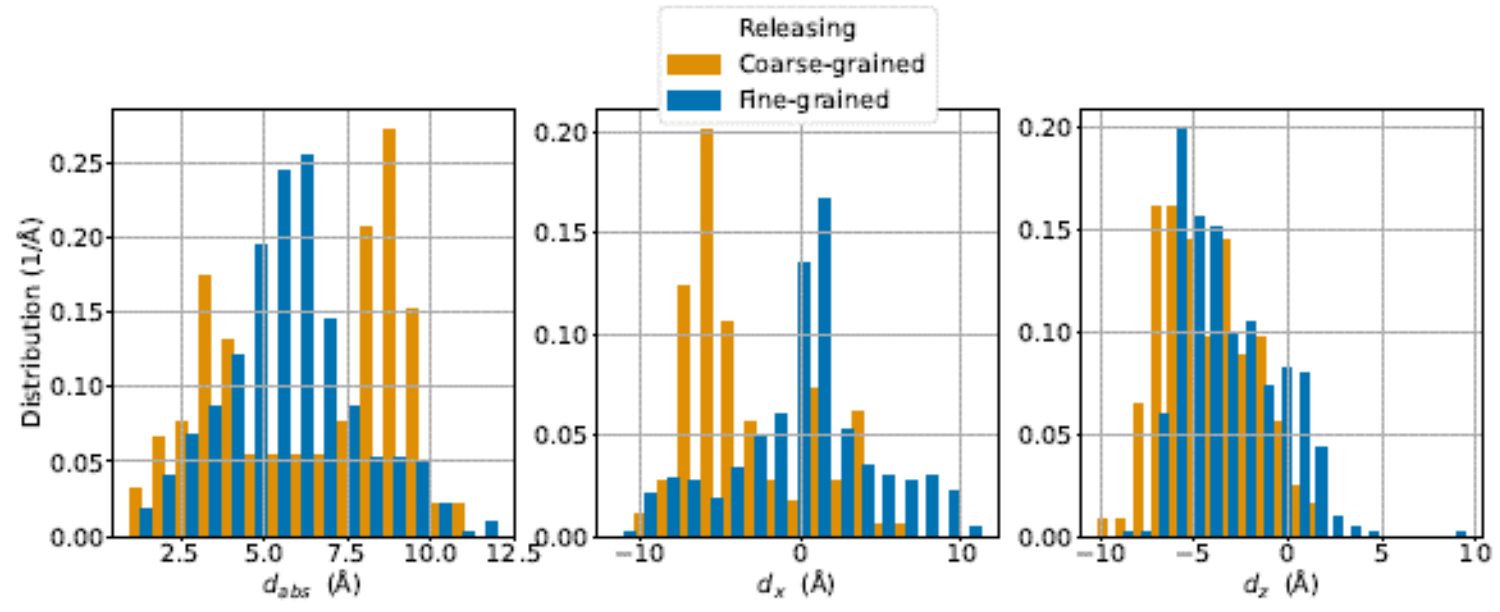

Figure 7

Normalized distributions of the center-of-mass displacements of grains during the (a) pressing, (b) holding, and (c) release phase. 


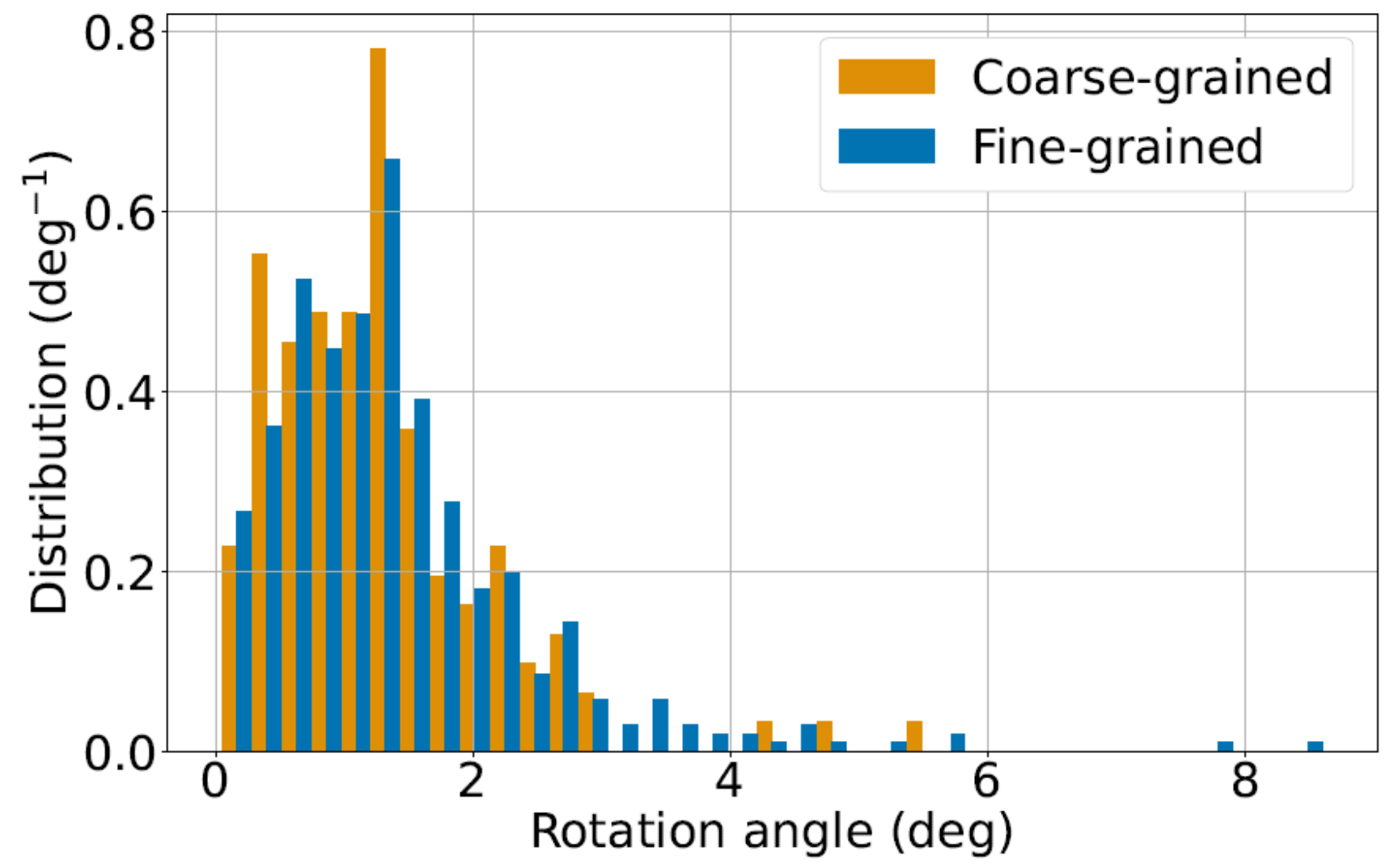

Figure 8

Distribution of grain rotation angles during the pressing phase. 


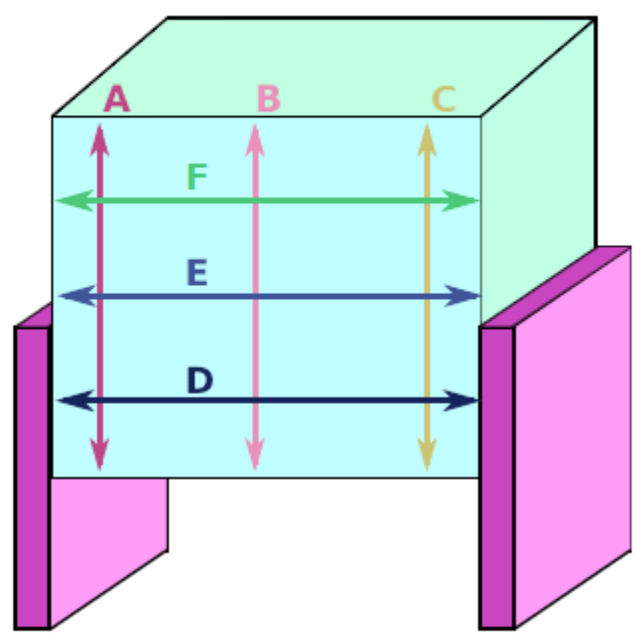

(a)

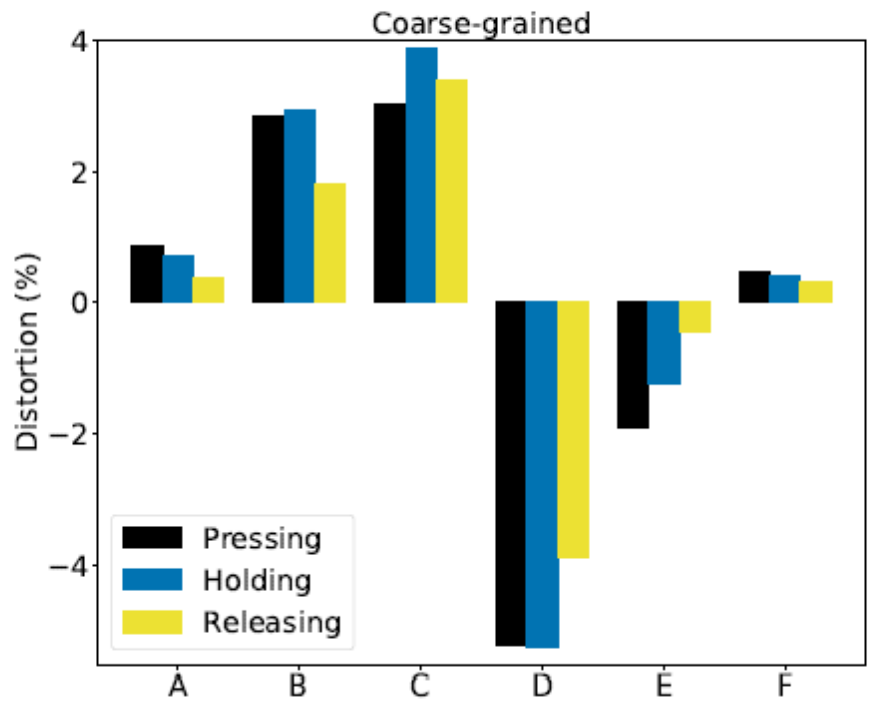

(b)

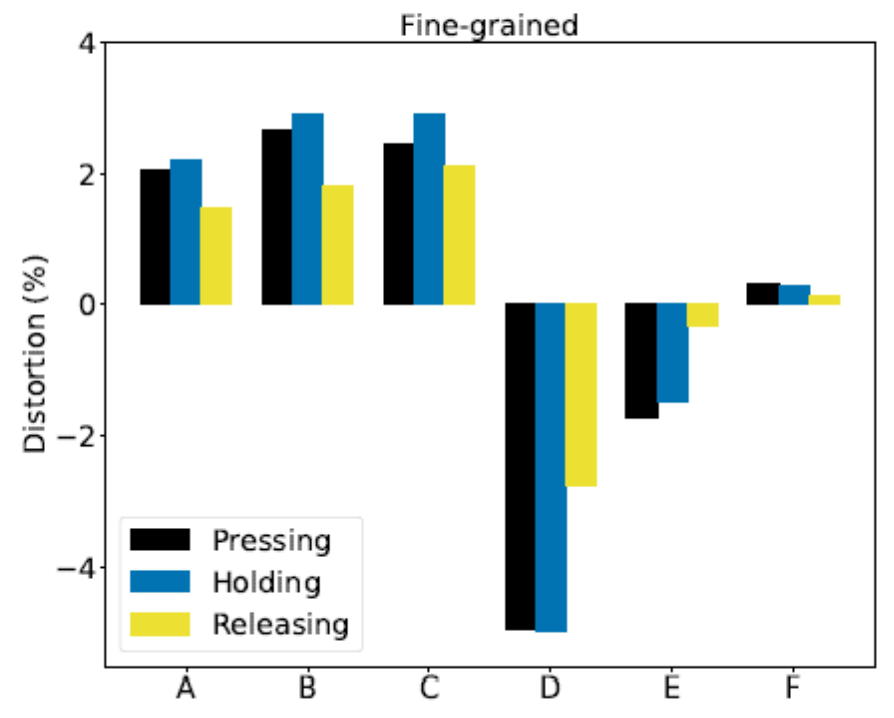

(c)

Figure 9

(a) Schematics indicating the positions where vertical $(A-C)$ and horizontal $(F-D)$ sample distortions were measured. (b) and (c) show simulation results for distortions for the coarse-grained (b) and finegrained (c) sample in the pressing, holding and release phase.

\section{Supplementary Files}

This is a list of supplementary files associated with this preprint. Click to download.

- subm.tex 